\title{
Numerical evaluation of cardiac mechanical markers as estimators of the electrical activation time
}

\author{
P. Colli Franzone ${ }^{*} \quad$ L. F. Pavarino ${ }^{\dagger} \quad$ S. Scacchi $^{\dagger}$
}

\begin{abstract}
Recent advances in the development of non-invasive cardiac imaging technologies have made it possible to measure longitudinal and circumferential strains at a high spatial resolution also at intramural level. Local mechanical activation times derived from these strains can be used as non-invasive estimates of electrical activation, in order to determine e.g. the origin of premature ectopic beats during focal arrhythmias or the pathway of reentrant circuits. The aim of this work is to assess the reliability of mechanical activation time markers derived from longitudinal and circumferential strains, denoted by $\mathrm{AT}_{e l l}$ and $\mathrm{AT}_{e c c}$, respectively, by means of three-dimensional cardiac electromechanical simulations. These markers are compared against the electrical activation time $\left(\mathrm{AT}_{v}\right)$, computed from the action potential waveform, and the reference mechanical activation markers derived from the active tension and fiber strain waveforms, denoted by $\mathrm{AT}_{t a}$ and $\mathrm{AT}_{\text {eff }}$, respectively. Our numerical simulations are based on a strongly coupled electromechanical model, including Bidomain representation of the cardiac tissue, mechano-electric (i.e. stretch-activated channels) and geometric feedbacks, transversely isotropic strain energy function for the description of passive mechanics and detailed membrane and excitation-contraction coupling models. The results have shown that, during endocardial and epicardial ectopic stimulations, all the mechanical markers considered are highly correlated with $\mathrm{AT}_{v}$, exhibiting correlation coefficients larger than 0.8. However, during multiple endocardial stimulations, mimicking the ventricular sinus rhythm, the mechanical markers are less correlated with the electrical activation time, because of the more complex resulting excitation sequence. Moroever, the inspection of the endocardial and epicardial isochrones has shown that the $\mathrm{AT}_{\text {ell }}$ and $\mathrm{AT}_{\text {ecc }}$ mechanical activation sequences reproduce only some qualitative features of the electrical activation sequence, such as the areas of early and late activation, but in some cases they might yield wrong excitation sources and significantly different isochrones patterns.
\end{abstract}

Keywords: cardiac electro-mechanical coupling; electrical and mechanical activation time; electro-mechanical delay; finite elasticity; Bidomain model; parallel numerical simulations.

\section{Introduction}

Nowadays, the procedure widely used for surgical treatment of cardiac arrhythmias via ablation is highly invasive, time-consuming and operator dependent, because it consists of a detailed mapping

${ }^{*}$ Dipartimento di Matematica, Università di Pavia, Via Ferrata 1, 27100 Pavia, Italy. E-mail: colli@imati.cnr.it. This work was partially supported by grants of CINECA and of the Istituto di Matematica Applicata e Tecnologie Informatiche, Pavia, Italy

${ }^{\dagger}$ Dipartimento di Matematica, Università di Milano, Via Saldini 50, 20133 Milano, Italy. E-mail: luca.pavarino@unimi.it, simone.scacchi@unimi.it. This work was partially supported by grants of Progetto G.N.C.S. of the Istituto Nazionale di Alta Matematica, Roma, Italy 
of electrical activation to determine the source and pathway of arrhythmia. Consequently, several research studies have been devoted to the development of effective non-invasive technologies for estimating the cardiac electrical activation with the aim of identifying the ablation sites. A noninvasive method to determine the arrhythmic sources and pathways is cardiac electrocardiography, based on the solution of the inverse problem of electrocardiology, see e.g. [62].

At the end of diastolic phase, the cardiac electrical activation induces cardiac muscle contraction and the associated deformation of the cardiac wall. The timing of mechanical activation follows the timing of electrical activation through the excitation-contraction coupling, which relates the membrane depolarization to myofilament activation and tension development, and gives rise to a fairly synchronous contraction see e.g. [47]. Mapping the cardiac contraction times in the normal human heart may give valuable insight into normal contraction patterns and may serve as a reference for interpreting data of patients considered for cardiac resynchronization therapy (CRT) and patients with ischemia. Contraction time maps can also be useful in order to estimate the fractional shortening of regional endocardial surface at the end systole for the characterization of chronic myocardial infarction, see e.g. [28].

In order to quantify the global and regional left ventricular function, several techniques to assess myocardial deformation and local myocardial strain parameters have been developed, see e.g. [64, $63,4,65,56]$. Previous experimental studies have partially validated different mechanical activation time markers as estimates of local electrical activation time in both healthy and pathological hearts, see $[4,3]$. Recently, a novel non-invasive ultrasound based imaging method named electromechanical wave imaging (EWI) has been developed, providing measurements of local displacement evolution, i.e. deformation, of myocardium after electrical activation,see e.g. [49, 50, 48, 19]. Strains can be computed from high resolution displacements, and then the mechanical activation sequence can be evaluated by defining suitable markers of mechanical activation derived from strain waveforms. Feasibility studies have compared EWI maps with the usual map of the electrical excitation sequence using animal experiments, see e.g. [19, 29]. These studies have shown that the EWI map is able to identify the site of stimulus sources and to map reentrant excitation circuits. However, due to the difficulty of detecting simultaneously electrical potential and displacements in vivo, these experimental validation studies have been limited to low spatial resolution.

In recent years, several research groups have performed cardiac computational studies based on three-dimensional electro-mechanical models, see [59, 40, 7, 9, 34, 2, 17, 61, 1, 27, 39, 14, 51, 38]. Numerical simulations might be an effective tool to validate mechanical activation markers at high spatial resolution, because cardiac electromechanical models provide information both on transmembrane electrical potential and displacement. Previous computational studies on this regard have mainly considered weakly coupled electromechanical models and Monodomain representation of the electrical current flow through the cardiac tissue.

Despite the large number of electro-mechanical simulation studies, only a few works $[60,35,36$, $30,17,31$ ] have investigated the complex spatio-temporal relationships and differences between the electric cardiac excitation sequence and the subsequent pattern of mechanical myocardial contraction and the cardiac electromechanical delay. We remark that the development of local myocyte active tension is delayed with respect to the local electric activation due to the excitation-contraction coupling. Moreover, strain development throughout the tissue is affected not only from the local active tension but also from the non-local passive mechanical behavior of myocardium. Evaluating the effects of such distortions on mechanical activation markers is important to assess their reliability as estimators of the electrical excitation sequence. 
The novelty of the present study is to provide a quantitative analysis, based on electro-mechanical numerical simulations, of the reliability of mechanical activation markers derived from longitudinal and circumferential strains, that can be measured in vivo. They are compared against the electrical activation time, computed from the action potential waveform, and the reference mechanical activation markers, computed from the active tension and fiber strain waveforms, that can not be measured in vivo at a high resolution. We also investigate the correlations and differences between electrical activation and mechanical contraction patterns under different stimulation protocols.

The numerical simulations presented are based on the strongly coupled electro-mechanical model developed in our previous works $[13,14]$, which takes into account the Bidomain representation of the cardiac tissue, mechano-electric (i.e. stretch-activated channels) and geometric feedbacks, transversely isotropic strain energy function for the description of passive mechanics and detailed membrane and excitation-contraction coupling models. The outcomes of our electro-mechanical solver, in particular in terms of strain waveforms and pressure-volume loop, have been validated by a comparison with experimental data in our previous works $[26,20]$.

\section{Methods}

\subsection{The cardiac electro-mechanical coupling model}

The strongly coupled electromechanical model that we adopt to describe the cardiac function is composed by four submodels, two for the mechanical activity and two for the electrical one.

a) Mechanical model of passive cardiac tissue. Denoting by $\Omega(t)$ the deformed cardiac domain at time $t$, we model the cardiac tissue as a non-linear hyperelastic material in the quasisteady state regime. Thus, neglecting gravitational forces, the Cauchy stress tensor satisfies the following equilibrium equation

$$
\operatorname{div} \sigma=0, \quad \mathbf{x} \in \Omega(t) .
$$

According to the active stress approach, the Cauchy stress tensor $\sigma$ is given by the sum of passive (pas), volumetric (vol) and active (act) components, i.e.

$$
\sigma=\sigma^{p a s}+\sigma^{v o l}+\sigma^{a c t}
$$

The material coordinates of the undeformed cardiac domain $\widehat{\Omega}$ are denoted by $\mathbf{X}=\left(X_{1}, X_{2}, X_{3}\right)^{T}$, the spatial coordinates of the deformed cardiac domain $\Omega(t)$ at time $t$ by $\mathbf{x}=\mathbf{x}(\mathbf{X}, t)$, the deformation gradient by $\mathbf{F}(\mathbf{X}, t)=\frac{\partial \mathbf{x}}{\partial \mathbf{X}}$ and the displacement field by $\mathbf{u}(\mathbf{X}, t)=\mathbf{x}-\mathbf{X}$.

Then the passive and volumetric components in (1) are defined as

$$
\sigma=J^{-1} \mathbf{F}\left(\frac{\partial W^{p a s}(\mathbf{E})}{\partial \mathbf{E}}+\frac{\partial W^{v o l}(\mathbf{E})}{\partial \mathbf{E}}\right) \mathbf{F}^{T},
$$

where $\mathbf{E}=\frac{1}{2}(\mathbf{C}-\mathbf{I})$ is the Green-Lagrange strain tensor, $\mathbf{C}=\mathbf{F}^{T} \mathbf{F}$ is the Cauchy - Green deformation tensor. Moreover, $W^{\text {pas }}$ is the exponential strain energy function modeling the passive mechanical behavior of myocardium as a transversely isotropic hyperelastic material. Its analytical expression is derived from the orthotropic law proposed in $[32,23])$. We added a volume change penalization term $W^{v o l}=K(J-1)^{2}$ in order to model the myocardium as a nearly incompressible material; here $K$ is a positive bulk modulus and $J=\operatorname{det} \mathbf{F}$. 
b) Mechanical model of active tension. The ventricles contraction results from the active tension generated by the myofilaments dynamics activated by calcium. We assume that the generated active tension acts only along the fiber direction, so that the active Cauchy stress is given by

$$
\sigma^{a c t}(\mathbf{x})=T_{a}(\mathbf{x}, t) \mathbf{a}_{f}(\mathbf{x}) \otimes \mathbf{a}_{f}(\mathbf{x}) .
$$

Here $\mathbf{a}_{f}$ is a unit vector parallel to the local fiber direction and $T_{a}$ is the active tension (fiber stress) related to the deformed domain.

In the Lagrangian framework, after the pull-back on the reference configuration $\widehat{\Omega}$, we have the equilibrium equation

$$
\operatorname{Div}(\mathbf{F S})=\mathbf{0} \quad \mathbf{X} \in \widehat{\Omega},
$$

where the second Piola-Kirchhoff stress tensor $\mathbf{S}$ is given by the sum of passive (pas), volumetric $(v o l)$ and active (act) components, i.e.

$$
\mathbf{S}=\mathbf{S}^{\text {pas, vol }}+\mathbf{S}^{a c t}
$$

with

$$
\mathbf{S}^{\text {pas }, v o l}=\frac{\partial W^{\text {pas }}(\mathbf{E})}{\partial \mathbf{E}}+\frac{\partial W^{v o l}(\mathbf{E})}{\partial \mathbf{E}}
$$

and

$$
\mathbf{S}^{a c t}(\mathbf{X}, t)=J \mathbf{F}^{-1} \sigma^{a c t} \mathbf{F}^{-T}=J \widehat{T_{a}}(\mathbf{X}, t) \frac{\widehat{\mathbf{a}}_{f} \otimes \widehat{\mathbf{a}}_{f}}{\widehat{\mathbf{a}}_{f}^{T} \mathbf{C} \widehat{\mathbf{a}}_{f}} .
$$

Here, $\widehat{T_{a}}(\mathbf{X}, t)=T_{a}((\mathbf{x}(\mathbf{X}, t), t)$ and we have written the unit vector parallel to the local fiber $\mathbf{a}_{f}$ in the deformed configuration in terms of the unit vector of fiber direction in the reference configuration $\widehat{\mathbf{a}}_{f}$, i.e.

$$
\mathbf{a}_{f}(\mathbf{x}(\mathbf{X}, t))=\frac{\mathbf{F} \widehat{\mathbf{a}}_{f}}{\left\|\mathbf{F} \widehat{\mathbf{a}}_{f}\right\|}=\frac{\mathbf{F} \widehat{\mathbf{a}}_{f}}{\sqrt{\widehat{\mathbf{a}}_{f}^{T} \mathbf{C} \widehat{\mathbf{a}}_{f}}},
$$

from which it follows

$$
\mathbf{a}_{f} \otimes \mathbf{a}_{f}=\frac{\mathbf{F} \widehat{\mathbf{a}}_{f} \otimes \mathbf{F} \widehat{\mathbf{a}}_{f}}{\left\|\mathbf{F} \widehat{\mathbf{a}}_{f}\right\|^{2}}=\frac{\mathbf{F} \widehat{\mathbf{a}}_{f} \widehat{\mathbf{a}}_{f}^{T} \mathbf{F}^{T}}{\widehat{\mathbf{a}}_{f}^{T} \mathbf{C} \widehat{\mathbf{a}}_{f}} .
$$

The biochemically generated active tension $\widehat{T_{a}}(\mathbf{X}, t)$ depends on the intracellular calcium concentration $C a_{i}$, the fiber stretch $\lambda=\sqrt{\widehat{\mathbf{a}}_{f}^{T} \mathbf{C} \widehat{\mathbf{a}}_{f}}$, and the stretch-rate $\frac{d \lambda}{d t}$ along the fiber direction

$$
\widehat{T_{a}}(\mathbf{X}, t)=T_{a}\left(C a_{i}(\mathbf{X}, t), \lambda(\mathbf{X}, t), \frac{d \lambda}{d t}(\mathbf{X}, t)\right),
$$

and it evolves according to an active tension generation model based on calcium kinetic and myofilament dynamics, see e.g. [33, 41, 52]. In this work, we consider the model proposed in [33], updated in [41] and recently modified in Land et al. [37].

c) Bioelectrical model of cardiac tissue: the Bidomain model. In [13], we have shown how, starting from the charge conservation law on the deformed configuration, imposing the quasistatic regime and disregarding ionic diffusion with respect to drift current, the Bidomain model, usually written on a fixed domain, can be extended to a moving domain. Denoting by $v, u_{e}, \mathbf{w}, \mathbf{c}$ the 
transmembrane potential, the extracellular potential, the gating and ionic concentrations variables on the deformed configuration and by $\widehat{v}, \widehat{u} e, \widehat{\mathbf{w}}, \widehat{\mathbf{c}}$ the same quantities on reference configuration, the parabolic-elliptic formulation of the Bidomain model can be written on the deformed configuration $\Omega(t)$ as

$$
\left\{\begin{array}{l}
c_{m} \frac{\partial v}{\partial t}-\operatorname{div}\left(D_{i} \nabla\left(v+u_{e}\right)\right)+i_{i o n}(v, \mathbf{w}, \mathbf{c}, \lambda)=i_{a p p}^{i} \\
-\operatorname{div}\left(D_{i} \nabla v\right)-\operatorname{div}\left(\left(D_{i}+D_{e}\right) \nabla u_{e}\right)=i_{a p p}^{i}+i_{a p p}^{e}
\end{array}\right.
$$

with $c_{m}$ and $i_{\text {ion }}$ the membrane capacitance and ionic current per unit volume, respectively. In order to satisfy the compatibility condition $\int_{\Omega(t)}\left(i_{a p p}^{i}+i_{a p p}^{e}\right) d \mathbf{x}=0$, we choose $i_{a p p}^{i}=-i_{a p p}^{e}=i_{a p p}$. These two partial differential equations (PDEs) are coupled through the reaction term $i_{\text {ion }}$ with the ordinary differential equations (ODEs) system of the membrane model, given in $\Omega(t) \times(0, T)$ by

$$
\frac{\partial \mathbf{w}}{\partial t}-\mathbf{R}_{w}(v, \mathbf{w})=0, \quad \frac{\partial \mathbf{c}}{\partial t}-\mathbf{R}_{c}(v, \mathbf{w}, \mathbf{c})=0 .
$$

Assuming transversely isotropic properties of the intra- and extracellular media, the conductivity tensors on the deformed configuration are given by

$$
D_{i, e}=\sigma_{t}^{i, e} I+\left(\sigma_{f}^{i, e}-\sigma_{t}^{i, e}\right) \mathbf{a}_{f} \otimes \mathbf{a}_{f},
$$

where $\sigma_{f}^{i, e}, \sigma_{t}^{i, e}$ are the conductivity coefficients of the intra- and extracellular media measured along the fiber direction $\mathbf{a}_{f}$ and any cross fiber direction, respectively.

In the Lagrangian framework, after the pull-back on the reference configuration $\widehat{\Omega} \times(0, T)$, the Bidomain system (5) becomes

$$
\left\{\begin{array}{l}
c_{m} J\left(\frac{\partial \widehat{v}}{\partial t}-\mathbf{F}^{-T} \operatorname{Grad} \widehat{v} \cdot \mathbf{V}\right)-\operatorname{Div}\left(J \mathbf{F}^{-1} \widehat{D}_{i} \mathbf{F}^{-T} \operatorname{Grad}\left(\widehat{v}+\widehat{u}_{e}\right)\right)+J i_{i o n}(\widehat{v}, \widehat{\mathbf{w}}, \widehat{\mathbf{c}}, \lambda)=\widehat{J i_{a p p}}, \\
-\operatorname{Div}\left(J \mathbf{F}^{-1} \widehat{D}_{i} \mathbf{F}^{-T} \operatorname{Grad} \widehat{v}\right)-\operatorname{Div}\left(J \mathbf{F}^{-1}\left(\widehat{D}_{i}+\widehat{D}_{e}\right) \mathbf{F}^{-T} \operatorname{Grad} \widehat{u}_{e}\right)=0,
\end{array}\right.
$$

where $\mathbf{V}=\frac{\partial \mathbf{u}}{\partial t}$ is the rate of deformation; see [13] for the detailed derivation.

From (4), it follows that the tensors $D_{i, e}(x, t)$ written in the reference configuration are

$$
\widehat{D}_{i, e}(\mathbf{X}, t)=D_{i, e}(\mathbf{x}(\mathbf{X}, t), t)=\sigma_{t}^{i, e} I+\left(\sigma_{f}^{i, e}-\sigma_{t}^{i, e}\right) \frac{\mathbf{F} \widehat{\mathbf{a}}_{f} \widehat{\mathbf{a}}_{f}^{T} \mathbf{F}^{T}}{\widehat{\mathbf{a}}_{f}^{T} \mathbf{C} \widehat{\mathbf{a}}_{f}^{T}} .
$$

Therefore, the equivalent conductivity tensors appearing into the Bidomain model (7) written in the reference configuration are given by

$$
J \mathbf{F}^{-1} \widehat{D}_{i, e}(\mathbf{X}, t) \mathbf{F}^{-T}=\sigma_{t}^{i, e} \mathbf{C}^{-1}+\left(\sigma_{f}^{i, e}-\sigma_{t}^{i, e}\right) \frac{\widehat{\mathbf{a}}_{f} \widehat{\mathbf{a}}_{f}^{T}}{\widehat{\mathbf{a}}_{f}^{T} \mathbf{C} \widehat{\mathbf{a}}_{f}^{T}}
$$

The bioelectrical system (7-6) is completed by prescribing initial conditions on $\widehat{v}, \mathbf{w}$, c, insulating boundary conditions on $\widehat{u}_{e}, \widehat{u}_{i}=\widehat{v}+\widehat{u}_{e}$, and the intra- and extracellular applied current $\widehat{i}_{\text {app }}=\widehat{i}_{\text {app }}^{i}=$ $-\widehat{i}_{\text {app }}^{e}$. Since the extracellular potential $\widehat{u}_{e}$ is defined up to a time dependent constant in space $R(t)$ determined by the choice of the reference potential, we consider as a reference potential the average of the extracellular potential over the cardiac volume, i.e. we impose $\int_{\widehat{\Omega}} \widehat{u}_{e}(\mathbf{X}, t) J(\mathbf{X}, t) d \mathbf{X}=0$. 


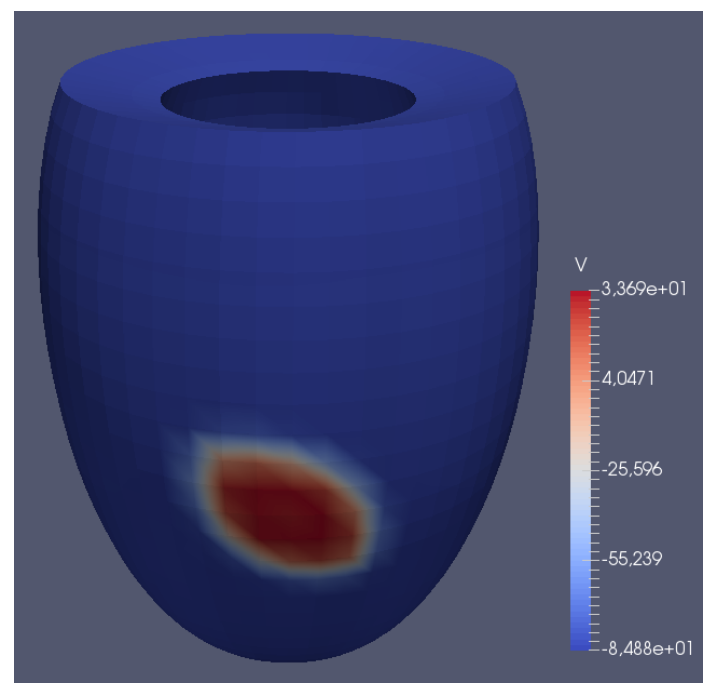

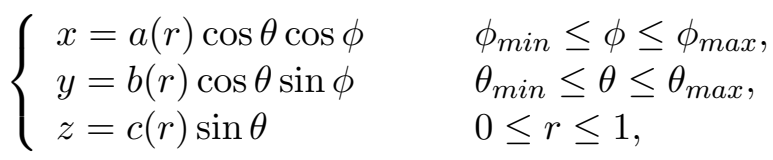

where:

$$
\begin{aligned}
& a(r)=a_{1}+r\left(a_{2}-a_{1}\right), \\
& b(r)=b_{1}+r\left(b_{2}-b_{1}\right), \\
& c(r)=c_{1}+r\left(c_{2}-c_{1}\right), \\
& a_{1}=b_{1}=1.5, a_{2}=b_{2}=2.7, c_{1}=4.4, c_{2}=5(\mathrm{~cm}) \\
& \phi_{\min }=-\frac{\pi}{2}, \phi_{\max }=\frac{3 \pi}{2}, \theta_{\min }=-\frac{3 \pi}{8}, \theta_{\max }=\frac{\pi}{8} .
\end{aligned}
$$

Figure 1: Truncated ellipsoidal domain modeling the left ventricle (left) and associated ellipsoidal coordinates (right).

d) Ionic membrane model and stretch-activated channel current. The ionic current in the Bidomain model ( 7$)$ is given by $i_{i o n}=\chi I_{i o n}$, where $\chi$ is the membrane surface to volume ratio and the ionic current per unit area of the membrane surface. $I_{i o n}$ is given by the sum $I_{i o n}(v, \mathbf{w}, \mathbf{c}, \lambda)=I_{i o n}^{m}(v, \mathbf{w}, \mathbf{c})+I_{s a c}$ of the ionic term $I_{i o n}^{m}(v, \mathbf{w}, \mathbf{c})$ given by the Faber-Rudy model (FR00) [24] (see also models.cellml.org/cellml), and the stretch-activated current $I_{\text {sac }}$ proposed in [42]; see also [16] for further details. The FR00 ionic model also specifies the functions $R_{w}(v, \mathbf{w})$ and $R_{c}(v, \mathbf{w}, \mathbf{c})$ in the ODE system (6), consisting of 25 equations modeling the dynamics of the ionic currents.

\subsection{Numerical methods, parameters calibration and simulations setup}

Domain geometry. We consider as cardiac domain $\widehat{\Omega}=\Omega(0)$ the image of a cartesian periodic slab using ellipsoidal coordinates, namely the left ventricular geometry is modeled as a truncated ellipsoid modeling as shown in Fig. 1. We will refer to the inner surface of the truncated ellipsoid $(r=0)$ as endocardium and to the outer surface $(r=1)$ as epicardium. The domain size corresponds to the left ventricle of a canine heart.

Cardiac fibers structure. The fibers rotate intramurally linearly with the depth for a total amount of $120^{\circ}$ proceeding counterclockwise from epicardium to endocardium. More precisely, in a local ellipsoidal reference system $\left(\mathbf{e}_{\phi}, \mathbf{e}_{\theta}, \mathbf{e}_{r}\right)$, the fiber direction $\mathbf{a}_{f}(\mathbf{x})$ at a point $\mathbf{x}$ is given by $\mathbf{a}_{f}(\mathbf{x})=\mathbf{b}_{f}(\mathbf{x}) \cos (\beta)+\mathbf{n}(\mathbf{x}) \cos (\beta)$, where

$$
\mathbf{b}_{f}(\mathbf{x})=\mathbf{e}_{\phi} \cos \alpha(r)+\mathbf{e}_{\theta} \sin \alpha(r),
$$

with

$$
\alpha(r)=\frac{2}{3} \pi(1-r)-\frac{\pi}{4}, \quad 0 \leq r \leq 1,
$$

where $\mathbf{n}(\mathbf{x})$ is the unit outward normal to the ellipsoidal surface at $\mathbf{x}$ and $\beta$ is the imbrication angle calibrated as in [11] given by $\beta=\arctan (\cos \alpha \tan \gamma)$, with $\gamma=\theta(1-r) 60 / \pi$. 
Space discretization. We discretize the cardiac domain with a hexahedral structured grid $T_{h_{m}}$ for the mechanical model (2) and $T_{h_{e}}$ for the Bidomain model (7), where $T_{h_{e}}$ is a refinement of $T_{h_{m}}$, i.e. $h_{m}$ is an integer multiple of $h_{e}$. We consider the variational formulations of both mechanical and bioelectrical models and then approximate all scalar and vector fields by isoparametric $Q_{1}$ finite elements in space. In all the electromechanical simulations, we employ an electrical mesh size $h_{e}=0.01 \mathrm{~cm}$ in order to properly resolve the sharp excitation front, while the smoother mechanical deformation allows us to use a coarse mechanical mesh of size $h_{m}=0.08 \mathrm{~cm}$. The resulting electrical mesh consists of $N_{\phi} \times N_{\theta} \times N_{k}=384 \times 192 \times 48$ elements, yielding about $3.63 \cdot 10^{6}$ nodes, while the mechanical mesh consists of $48 \times 24 \times 6$ elements, yielding about 8400 nodes.

Time discretization. The time discretization of the electromechanical model is performed by the following semi-implicit splitting method, where the electrical and mechanical time steps could be different. At each time step, we solve the following three subproblems.

a) Ionic update. Given $v^{n}, w^{n}, c^{n}$ at time $t_{n}$, solve the ODE system of the membrane model (6) with a first order implicit-explicit (IMEX) method to compute the new $w^{n+1}, c^{n+1}$.

b) Mechanical update. Given the calcium concentration $C a_{i}^{n+1}$, which is included in the concentration variables $c^{n+1}$, solve the variational formulation of the mechanical problem (2) and the active tension system to compute the new deformed coordinates $\mathbf{x}^{n+1}$, providing the new deformation gradient tensor $\mathbf{F}_{n+1}$. More in detail, denoting by $\mathbf{V}^{h}$ the finite element space for the displacement field, the discrete mechanical problem reads: find the displacement $\mathbf{u} \in \mathbf{V}^{h}$ such that, $\forall \mathbf{v} \in \mathbf{V}^{h}$,

$$
\int_{\widehat{\Omega}} \mathbf{F S}(\mathbf{u}): \operatorname{Grad} \mathbf{v}=0
$$

with $\mathbf{F}=\operatorname{Grad} \mathbf{u}$. The $k$-Newton iteration of the non-linear system (9) reads: find $\delta \mathbf{u} \in \mathbf{V}^{h}$ such that, $\forall \mathbf{v} \in \mathbf{V}^{h}$,

$$
\int_{\widehat{\Omega}} \operatorname{Grad} \delta \mathbf{u} \mathbf{S}\left(\mathbf{u}^{k}\right): \operatorname{Grad} \mathbf{v}+\int_{\widehat{\Omega}} \mathbf{F}_{k}^{T} \operatorname{Grad} \mathbf{v}: \frac{\partial \mathbf{S}\left(\mathbf{u}^{k}\right)}{\partial \mathbf{C}_{k}}: \mathbf{F}_{k}^{T} \operatorname{Grad} \delta \mathbf{u}=-\int_{\widehat{\Omega}} \mathbf{F}_{k} \mathbf{S}\left(\mathbf{u}^{k}\right): \operatorname{Grad} \mathbf{v},
$$

with $\mathbf{F}_{k}=\operatorname{Grad} \mathbf{u}^{k}$ and $\mathbf{C}_{k}=\mathbf{F}_{k}^{T} \mathbf{F}_{k}$. Then set $\mathbf{u}^{k+1}=\mathbf{u}^{k}+\delta \mathbf{u}$. In order to avoid numerical instabilities, in point b) the finite elasticity equations (9) and the active tension model are solved in a coupled way using the update method proposed in [43], which consists of updating the variables of the active tension model at each Newton iteration together with the solution of the mechanical deformation; for detailed investigations on the numerical treatment of cardiac mechanical models, we refer e.g. to $[44,58]$.

c) Electrical update. Given $w^{n+1}, c^{n+1}, \mathbf{F}_{n+1}$ and $J_{n+1}=\operatorname{det}\left(\mathbf{F}_{n+1}\right)$, solve the variational formulation of the Bidomain system (7) with a first order IMEX method and compute the new electric potentials $v^{n+1}, u_{e}^{n+1}$ with an operator splitting method, consisting of decoupling the parabolic from the elliptic equation.

In our simulations, the electrical time step size is $\Delta_{e} t=0.05 \mathrm{~ms}$, while the mechanical times step is $\Delta_{m} t=0.25 \mathrm{~ms}$. In order to approximate the convective term in the variational formulation of (7), an upwind computation of the nodal gradient of $v^{n}$ is derived, projected onto the deformation rate $\frac{\mathbf{x}^{n+1}-\mathbf{x}^{n}}{\Delta t_{n}}$ vector and then integrated against the test function. We refer to [13] for more details about the numerical scheme.

Computational kernels and parallel solvers. Due to the employed space and time discretization strategies, at each time step, the main computational kernels are:

i) solving the non-linear system deriving from the discretization of the mechanical problem 
(2) by a parallel Newton-GMRES-Algebraic Multigrid method, see [12]; an alternative NewtonGMRES-BDDC solver could be used as well, see [46];

ii) solving the two linear systems deriving from the discretization of the elliptic and parabolic equations in the Bidomain model (7) by a parallel Conjugate Gradient method preconditioned with a Multilevel Additive Schwarz preconditioner studied in [45, 55].

Our parallel simulations have been performed on a Linux cluster using the parallel library PETSc [6] from the Argonne National Laboratory, while visualization of the results have been obtained with Paraview and Matlab software.

Parameters calibration. a) Mechanical model. The parameters of the transversely isotropic strain energy function are adapted from [23]. The bulk modulus is $K=200 \mathrm{kPa}$.

b) Active tension model. The active tension model and its parameters are as in Land et al. [37].

c) Bidomain model. The values of the transversely isotropic conductivity coefficients in (8), that we use in all the numerical tests, are $\sigma_{f}^{i}=3, \sigma_{t}^{i}=0.31525, \sigma_{f}^{e}=2, \sigma_{t}^{e}=1.3514$, all expressed in $m \Omega^{-1} \mathrm{~cm}^{-1}$. These values, coupled with the FR00 membrane model, predict conduction velocities of about 0.061 and $0.027 \mathrm{~cm} / \mathrm{ms}$ for excitation wavefronts propagating along and across the fiber direction, respectively. We remark that these conduction velocities are within the physiological range. The membrane surface to volume ratio is $\chi=10^{3} \mathrm{~cm}^{-1}$ and the membrane capacitance per unit volume is $c_{m}=\chi C_{m}$, where $C_{m}=1 \mu \mathrm{F} / \mathrm{cm}^{2}$ is the membrane capacitance per unit area.

d) FR00 membrane model. We consider the calibration of the FR00 model proposed in [24].

Initial and boundary conditions. The initial conditions for the electrical model are the resting values for all the potentials and gating variables of the FR00 model, while the boundary conditions for the Bidomain model are for insulated tissue. Regarding the mechanical boundary conditions, in order to prevent rigid body motion, we set to zero the total displacement vector at the circumferential basal line meeting the endocardial surface and the third displacement component on the rest of the basal surface. Moreover, on the endocardial surface we impose a Neumann boundary condition given by the intracavitary blood pressure, whose dynamics is modeled according to a simple pressure-volume loop model; see [23, 15].

Electrical and mechanical activation time markers. Mechanical activation is related to the moment of local tension development, reflecting the local myocytes contraction, hence to the rising phase of the active tension $T_{a}(t)$. Unfortunately, the local active tension can not be measured in in vivo heart tissue, but it is possible to measure strains along different directions, usually circumferential and longitudinal. Mechanical activation time markers are computed during the shortening phase of such strain waveforms. Numerous approaches have been used in the past to define mechanical activation markers, see [5, 47, 21, 64, 63, 25, 60, 53, 54, 4, 57, 30, 29].

Given a unit vector a, using the symmetric Green-Lagrange strain tensor, we compute the projected strain $\mathbf{a}^{T} \mathbf{E}(\mathbf{X}, t) \mathbf{a}$, which is a measure of the change of the deformation state at time $t$ with respect to the reference configuration, along the direction identified by $\mathbf{a}$. The previous strain measure is usually considered along different preferential directions, i.e. along the fiber direction $\mathbf{a}_{f}$ or along the circumferential (c), longitudinal (l) and radial (r) directions $\mathbf{a}_{s}$, with $s=c, l, r$, respectively.

We consider this measure of the strain change with respect to the deformed configuration of the end diastole (the moment when ejection starts), i.e. we compute $E_{s s}(\mathbf{X}, t):=\mathbf{a}_{s}^{T} \mathbf{E}(\mathbf{X}, t) \mathbf{a}_{s}-$ $\mathbf{a}_{s}^{T} \mathbf{E}\left(\mathbf{X}, t_{E D}\right) \mathbf{a}_{s}$, where $\mathbf{a}_{s}, s=f, l, c, r$ is the unit vector along the fiber, longitudinal, circumferential and radial directions, respectively.

In the literature, mechanical activation time markers at a given location $\mathbf{X}$ are introduced in 

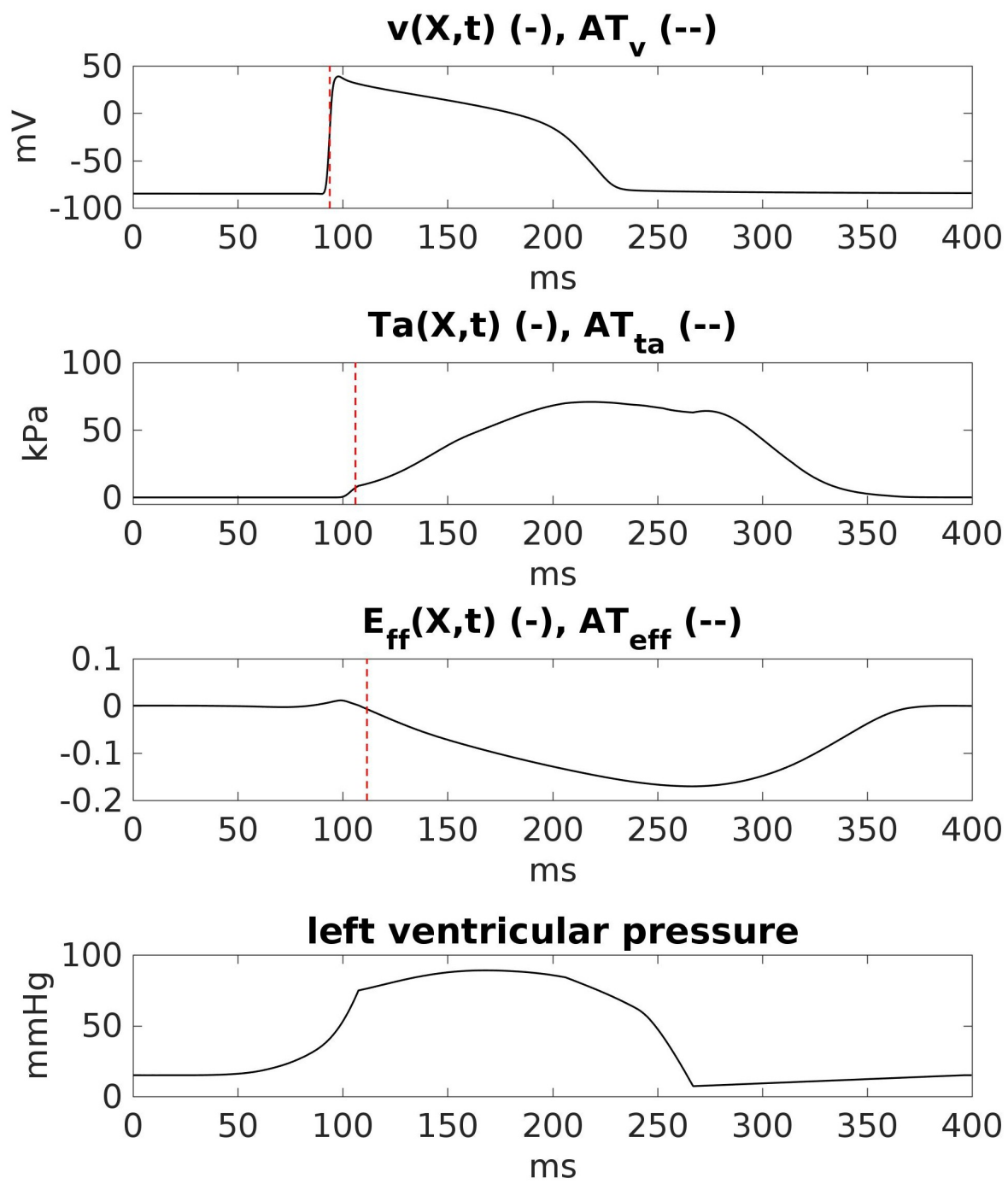

Figure 2: Examples of electrical and mechanical activation markers for waveforms associated with an epicardial basal point in the case of an ectopic endocardial stimulation. 
different ways by considering the strain temporal waveform $\mathbf{a}^{T} \mathbf{E}(\mathbf{X}, t) \mathbf{a}$. We recall that, depending on the pacing protocol, the simulated strain waveforms show different temporal patterns, such as: i) prestretched regions displaying a peak of lengthening, due to contraction of other region after the diastolic phase, followed by a shortening; ii) weakly and early shortening followed by stretching; iii) no stretching before shortening.

At selected nodes $\mathbf{X} \in \widehat{\Omega}$, we computed the following electrical activation time marker:

- $\operatorname{AT}_{v}(\mathbf{X})$, defined as the unique instant of maximum time derivative of the transmembrane potential $\widehat{v}(\mathbf{X}, t)$ during the upstroke phase of the action potential,

and four mechanical activation time markers:

- $\mathrm{AT}_{t a}(\mathbf{X})$, defined as the unique instant when the active tension $T_{a}$ increases above the threshold $10 \% \max _{t} T_{a}(\mathbf{X}, t)$;

- $\operatorname{AT}_{\text {eff }}(\mathbf{X})$, defined as the unique instant when, starting from the onset of myofiber shortening, the fiber strain $E_{f f}(\mathbf{X}, t)$ decreases below the threshold $\max _{t} E_{f f}(\mathbf{X}, t)-10 \%\left(\max _{t} E_{f f}(\mathbf{X}, t)-\right.$ $\left.\min _{t} E_{f f}(\mathbf{X}, t)\right)$;

- $\mathrm{AT}_{\text {ell }}(\mathbf{X})$, defined as the unique instant when, starting from the onset of myofiber shortening, the longitudinal strain $E_{l l}(\mathbf{X}, t)$ decreases below the threshold $\max _{t} E_{l l}(\mathbf{X}, t)-10 \%\left(\max _{t} E_{l l}(\mathbf{X}, t)-\right.$ $\left.\min _{t} E_{l l}(\mathbf{X}, t)\right)$

- $\operatorname{AT}_{e c c}(\mathbf{X})$, defined as the unique instant when, starting from the onset of myofiber shortening, the circumferential strain $E_{c c}(\mathbf{X}, t)$ decreases below the threshold $\max _{t} E_{c c}(\mathbf{X}, t)-$ $10 \%\left(\max _{t} E_{c c}(\mathbf{X}, t)-\min _{t} E_{c c}(\mathbf{X}, t)\right)$.

Figure 2 displays some representative electrical and mechanical waveforms and the left ventricular pressure waveform, in case of an ectopic endocardial stimulation. In our simulations, several strain waveforms do not show an initial peak followed by a shortening, thus we adopt as a mechanical activation time marker the one proposed in $[4,30]$, which seems to be more robust than other choices.

\section{Results}

\subsection{Endocardial ectopic beat simulation}

In this test, we apply an extracellular-intracellular stimulus, i.e. $i_{a p p}=i_{a p p}^{e}=-i_{a p p}^{i}$, on a small tissue volume located at the center of the anterior endocardial surface. The resulting anterior endocardial electric excitation sequence (see Fig. 3, first column) proceeds fast along the endocardial fiber direction oriented roughly at 75 degree, with isochrones presenting the typical flattened quasi-elliptical shape. The posterior endocardial electric excitation sequence shows that apical and lateral regions are activated earlier and displays a typical V-shaped pattern converging toward the latest activated basal regions. Moreover, due to the change of the wavefronts curvature, a fast propagation occurs along the vertical $\mathrm{V}$-shaped line as confirmed by the space between subsequent excitation fronts, according to the previous simulation results reported e.g. in [11].

All the mechanical activation sequences on the anterior endocardial surface share the same qualitative features of the $\mathrm{AT}_{v}$ sequence, characterized by a minimum approximatively located 
near the stimulation site. However, the $\mathrm{AT}_{\text {ell }}$ and $\mathrm{AT}_{e c c}$ isochrones loose the quasi-elliptical shape around the stimulation site. On the posterior endocardial surface (see Fig. 3 second row), only the $\mathrm{AT}_{t a}$ and $\mathrm{AT}_{e c c}$ markers display the $\mathrm{V}$-shaped pattern. We remark that $\mathrm{AT}_{\text {ell }}$ marker yields unreliable estimates near the apical region.

From a global point of view, i.e. comparing the markers patterns on the entire ventricular volume, the $\mathrm{AT}_{t a}$ sequence is highly correlated with the $\mathrm{AT}_{v}$ sequence, with a correlation coefficient (CC) amounting to 0.96. The three mechanical markers derived from the strain tensor $E$ are less correlated with, in fact the CC ranges between 0.85 ( $\left.\mathrm{AT}_{e c c} \mathrm{vs}_{\mathrm{AT}}\right)$ and $0.90\left(\mathrm{AT}_{\text {eff }} \mathrm{vs}_{\mathrm{AT}}\right)$.

The vertical line intercept of the regression line quantifies the average electro-mechanical delay between the two compared markers. From Fig. 4, we can estimate a delay of $35 \mathrm{~ms}$ for $\mathrm{AT}_{t a}, 14$ $\mathrm{ms}$ for $\mathrm{AT}_{\text {eff }}, 18 \mathrm{~ms}$ for $\mathrm{AT}_{\text {ell }}, 10 \mathrm{~ms}$ for $\mathrm{AT}_{e c c}$, all compared versus $\mathrm{AT}_{v}$.

The slope of the regression line is instead a measure of the electro-mechanical delay distribution. A slope larger than 1 indicates that the delay is larger in regions of late activation, while a slope smaller than 1 indicates that the delay is larger in regions of early activation. The slopes of the regression lines reported in Fig. 4 are 0.85 for $\mathrm{AT}_{t a}, 1.12$ for $\mathrm{AT}_{\text {eff }}, 0.95$ for $\mathrm{AT}_{\text {ell }}$ and 1.24 for $\mathrm{AT}_{\text {ecc }}$.

We report in Fig. 5 the fiber, longitudinal and circumferential strain waveforms at three selected endocardial anterior sites, located at the base, at the center and at the apex of the ventricular volume. We observe that most of these waveforms, after the associated time of local electrical activation, are monotone decreasing during the contraction phase, without or with a very small a prestretch dome. The $\mathrm{AT}_{e f f}$ and $\mathrm{AT}_{\text {ell }}$ markers are in good agreement, while the $\mathrm{AT}_{e c c}$ marker is delayed at all sites.

Then we report in Fig. 6 the distributions of the electro-mechanical delays $\mathrm{AT}_{t a}-\mathrm{AT}_{v}, \mathrm{AT}_{e f f}-\mathrm{AT}_{v}$, $\mathrm{AT}_{e l l}-\mathrm{AT}_{v}$ and $\mathrm{AT}_{e c c}-\mathrm{AT}_{v}$ on the endocardial surface. All the strain derived delays $\left(\mathrm{AT}_{e f f}-\mathrm{AT}_{v}\right.$, $\mathrm{AT}_{\text {ell }}-\mathrm{AT}_{v}$ and $\left.\mathrm{AT}_{e c c}-\mathrm{AT}_{v}\right)$ present patterns significantly different from the $\mathrm{AT}_{t a}-\mathrm{AT}_{v}$, that should be considered as the reference one.

We have also run a simulation in a transmural heterogeneous left ventricle, by modifying the IKs and ICaL currents, based on the data reported in the previous works $[18,8,10]$. In particular, we have subdivided the ventricular wall into three transmural layers of the same depth. The IKs current is scaled by 1, 0.7 and 1.7 in the sub-endo-, mid- and sub-epicardial layers, respectively. The ICaL current is scaled by 1, 1.5 and 1.5 in the sub-endo-, mid- and sub-epicardial layers, respectively. The stimulation protocol is the same of the homogeneous case, thus endocardial ectopic. The results (not shown) exhibit a markers performance comparable to the homogeneous simulation, with relative errors lower than $3 \%$ for all mechanical markers.

\subsection{Epicardial ectopic beat simulation}

In this simulation, an extracellular-intracellular stimulus is applied on a small tissue volume located at the center of the anterior epicardial surface. As a result, the anterior epicardial electrical activation sequence, reported in Fig. 7 (first column), related to the $A T_{v}$ marker, presents a minimum located at the stimulation point and quasi-elliptical isochrones with the major axis aligned as the local epicardial fiber direction, roughly oriented at $-45^{\circ}$. On the posterior epicardial side, the excitation sequence presents a typical V-shaped pattern, proceeding mainly from the apical to the latest activated basal regions. On the endocardium (see Fig. 8, first column), the anterior electric excitation sequence shows a minimum corresponding to the endocardial activation breakthrough and quasi-elliptical isochrones, more rounded than the epicardial ones, with the major axis 
aligned mainly along the local fiber direction, oriented roughly at 75 degree. From the breakthrough site, the excitation propagates fast in all directions as confirmed by the space between subsequent isochrones.

On the epicardial surface, all the mechanical markers, except $\mathrm{AT}_{\text {ell }}$, display a distribution similar to $\mathrm{AT}_{v}$, with a minimum located at the central anterior region where the stimulus has been applied and a $\mathrm{V}$-shaped pattern on the posterior side. The $\mathrm{AT}_{e c c}$ and $\mathrm{AT}_{\text {ell }}$ patterns show the appearance of a spurious excitation source located at the basal and apical region, respectively.

On the anterior endocardial surface, all mechanical markers present the same features of the $\mathrm{AT}_{v}$ sequence, characterized by a minimum located near the endocardial excitation breakthrough point. Instead, on the posterior side, only the $\mathrm{AT}_{t a}$ and $\mathrm{AT}_{e c c}$ markers reproduce well the $\mathrm{V}$ shaped pattern. However, $\mathrm{AT}_{\text {ecc }}$ exhibits a significantly delayed excitation sequence, reaching the ventricular base at about $280 \mathrm{~ms}$ compared to the $180 \mathrm{~ms}$ of $\mathrm{AT}_{\text {eff }}$ and $\mathrm{AT}_{\text {ell }}$. As in the previous simulation, we remark that the $\mathrm{AT}_{\text {ell }}$ marker yields unreliable estimates near the apical region.

The $\mathrm{AT}_{t a}$ pattern is highly correlated with the $\mathrm{AT}_{v}$ sequence, yielding a $\mathrm{CC}$ of 0.94 , see Fig. 9. Among the mechanical markers derived from the strain tensor $E$, the most correlated with $\mathrm{AT}_{v}$ is $\mathrm{AT}_{\text {eff }}(\mathrm{CC}=0.89)$. The vertical line intercept values of the regression lines are $34 \mathrm{~ms}$ for $\mathrm{AT}_{t a}$, $16 \mathrm{~ms}$ for $\mathrm{AT}_{\text {eff }}, 31 \mathrm{~ms}$ for $\mathrm{AT}_{\text {ell }},-1 \mathrm{~ms}$ for $\mathrm{AT}_{\text {ecc }}$. The slopes of the regression lines are: 0.86 for $\mathrm{AT}_{t a}, 1.12$ for $\mathrm{AT}_{e f f}, 0.86$ for $\mathrm{AT}_{\text {ell }}$ and 1.34 for $\mathrm{AT}_{e c c}$.

Different from the previous endocardial stimulation, almost all the endocardial strain waveforms reported in Fig. 10 show a prestretch dome before the contraction phase. Again the $\mathrm{AT}_{\text {eff }}$ and $\mathrm{AT}_{\text {ell }}$ markers are in good agreement, while the $\mathrm{AT}_{e c c}$ marker yields a reduced electro-mechanical delay in the basal and central sites. We also show in Fig. 11 the posterior endocardial strain waveforms at three selected locations. All these posterior waveforms present a significant prestretch dome before the contraction phase. Analogously to the anterior waveforms, the $\mathrm{AT}_{e c c}$ marker exhibits a reduced delay with respect to the electric activation time.

\subsection{Multiple endocardial stimulations}

In this case, excitation starts from several endocardial sites located mainly on the anterior apical side of the ventricle. The aim is to mimick the stimulation elicited from the Purkinjie Ventricular Junctions (PVJs). In this way, the resulting activation sequence behaves similarly to the one occurring during sinus rhythm, which in the left ventricle proceeds mainly from endocardium to epicardium, reaching earlier the apical regions and later the basal ones, see [22].

As a result of this stimulation setup, activation is slightly faster on the anterior endocardial surface than on the posterior one, see Fig. 12. Only the $\mathrm{AT}_{t a}$ and $\mathrm{AT}_{\text {eff }}$ patterns are able to identify the number and location of the source excitation sites. In fact, due to the complex resulting activation sequence, with many wavefront collisions, the performance of the mechanical markers in reproducing the $\mathrm{AT}_{v}$ excitation pattern is worse than in the ectopic stimulation cases, as confirmed by the low CC reported in Fig. 13. The vertical line intercept values of the regression lines are $37 \mathrm{~ms}$ for $\mathrm{AT}_{t a}, 12 \mathrm{~ms}$ for $\mathrm{AT}_{\text {eff }}, 9 \mathrm{~ms}$ for $\mathrm{AT}_{\text {ell }}, 63 \mathrm{~ms}$ for $\mathrm{AT}_{\text {ecc }}$. The slopes of the regression lines are: 0.61 for $\mathrm{AT}_{t a}, 1.28$ for $\mathrm{AT}_{\text {eff }}, 1.10$ for $\mathrm{AT}_{\text {ell }}$ and 1.42 for $\mathrm{AT}_{\text {ecc }}$.

The strain waveforms displayed in Fig. 14 confirm the good agreement, observed in the previous two simulations, between the $\mathrm{AT}_{\text {eff }}$ and $\mathrm{AT}_{\text {ell }}$ markers, while the $\mathrm{AT}_{\text {ecc }}$ marker is significantly delayed. 


\section{Discussion}

We have investigated by means of three-dimensional electromechanical simulations the reliability of mechanical activation time markers, derived from active stress $\left(\mathrm{AT}_{t a}\right)$ and fiber $\left(\mathrm{AT}_{e f f}\right)$, longitudinal $\left(\mathrm{AT}_{\text {ell }}\right)$ and circumferential $\left(\mathrm{AT}_{e c c}\right)$ strains, as estimates of the electrical activation time $\left(\mathrm{AT}_{v}\right)$, computed from the action potential waveform.

While local active stress and fiber strain can not be measured non-invasively in vivo, longitudinal and circumferential strains can be detected using recent non-invasive imaging technologies, see e.g. $[25,49,50,29]$, at a high resolution not only on the endocardial and epicardial surfaces but also through the transmural wall. Local mechanical activation times derived from these strains can be used as non-invasive estimates of electrical activation, e.g. to determine the origin of premature ectopic beats during focal arrhythmias or the pathway of reentrant circuits. In order to validate the reliability of these mechanical activation markers, simultaneous measurements of electrical and mechanical activation sequences should be performed in vivo, but current experimental technologies do not allow such measurements at a high spatial resolution covering the whole ventricular wall. Hence, at the moment, only detailed electromechanical models and simulations make it possible an in-depth validation of mechanical activation time markers, as done in the pioneering study [48].

Different from previous computational studies, mainly based on weakly coupled electromechanical Monodomain models, the numerical simulations performed in the present investigation are based on a strongly coupled electromechanical model, including Bidomain representation of the cardiac tissue, mechano-electric (i.e. with stretch-activated channels) and geometric feedbacks, transversely isotropic strain energy function for the description of passive mechanics and detailed membrane and excitation-contraction coupling models.

We have studied the performance of four mechanical activation markers by considering three different stimulation protocols yielding different electrical activation sequences: ectopic endocardial stimulation, ectopic epicardial stimulation and multiple sites endocardial stimulation, mimicking the sinus rhythm triggered by PVJs.

The results have shown that, during endocardial and epicardial ectopic stimulations, all the mechanical markers are highly correlated with $\mathrm{AT}_{v}(\mathrm{CC}>0.8) . \mathrm{AT}_{t a}$ presents the highest $\mathrm{CC}$ $(>0.9)$, whereas the three markers derived from strain are comparable, with CC ranging between 0.85 and 0.9. However, despite the high correlation, the regression plots displayed in Figures 4 and 9 show that the range of mechanical activation times associated to a specific electrical activation time is large, especially for the middle range of electrical activation times, in agreement with the experimental findings reported in [25], which only refer to epicardial circumferential strains. This result could be due to the fact that the ventricular regions in the middle range of electrical activation times are larger than the regions of early and late activation, thus they exhibit a more heterogeneous mechanical behavior. Moreover, the slightly worse performance of the strain-derived mechanical markers with respect to $\mathrm{AT}_{t a}$ could be attributed to the influence of non-local passive mechanical behavior on the strain waveforms. Indeed, although the shortening phase of the strain waveforms $E_{f f}, E_{c c}$ and $E_{l l}$ reflects the local mechanical contraction, it is also affected from the loads imposed on adjacent regions and by the global heterogeneous tissue deformation. $\mathrm{AT}_{t a}$ is instead more related to the local mechanical contraction, because it depends directly on the local intracellular calcium evolution.

During multiple endocardial stimulations, the mechanical markers are less correlated with the electrical activation time $(\mathrm{CC} \leq 0.82)$, especially $\mathrm{AT}_{e c c}(\mathrm{CC}=0.5)$. This poor performance in case of multiple endocardial stimulations has been also observed experimentally in the previous work 
[29], even though the definition of mechanical activation is different. Indeed, Grondin et al. defined the mechanical activation time as the first instant of zero-crossing of the strain time derivative after QRS. In our simulated signals, many waveforms do not present a zero-crossing of the derivative slightly after the electrical activation, thus we have used the more robust definition of mechanical activation time reported in Sec. 2.2, and previously used by $[4,30]$.

The inspection of the endocardial and epicardial isochrones has shown that the mechanical activation sequences reproduce the same qualitative features of the electrical activation sequence, with the same location of early and late activation areas. However, the $\mathrm{AT}_{\text {ell }}$ and $\mathrm{AT}_{\text {ecc }}$ mechanical activation sequences display significant discrepancies with respect to the reference electrical excitation sequence, yielding spurious excitation sources and different isochrones morphology, especially in the case of multiple endocardial stimulations modeling the excitation triggered by PVJs.

Moreover, all mechanical activation sequences present a delay, the so-called electro-mechanical delay (EMD), with respect to the electrical one. We remark that the intercept of the regression lines displayed in Figures 9, 4 and 13 is an estimate of the associated EMD. In all the stimulation protocolos considered, the $\mathrm{AT}_{t a}$ and $\mathrm{AT}_{\text {eff }}$ markers exhibit intercept values ranging between 3437 and 12-16 ms, respectively, while the $\mathrm{AT}_{\text {ell }}$ and $\mathrm{AT}_{e c c}$ markers exhibit a much larger range. This indicates that the performance of the two latter markers in measuring EMD is much more affected from the stimulation protocol and the resulting electrical activation sequence. The slope of the regression lines indicates that EMD, especially the one associated with the $\mathrm{AT}_{\text {eff }}$ and $\mathrm{AT}_{\text {ecc }}$ markers, is larger in the regions of late activation, corresponding to the posterior and basal ones. These results are in agreement with those previously reported in [30].

Regarding the morphology of the simulated strain waveforms, we finally observe that the regions of late activation present longitudinal and circumferential strains waveforms with a large prestretch dome preceding the contraction phase, see Fig. 11. These observations, which hold on the entire ventricular volume and irrespectively of the stimulation protocol, agree with the experimental findings obtained in [25], related to an epicardial stimulation protocol and epicardial strain mapping.

\subsection{Limitations and future works}

We have considered an idealized left ventricular geometry instead of a realistic patient-specific biventricular geometry. We have also not included complex boundary conditions such as the contact with pericardium. A more realistic and detailed representation of PVJs could be also included as in [38]. Further heterogeneities in $C a^{2+}$ handling might affect the reliability of mechanical markers as well. Our choice to reduce the degree of complexity of modeling assumptions was done in order to better estimate the effective role of deformation on the mechanical activation markers, in particular on their spatial distribution. Future works should investigate the reliability of mechanical activation markers including further heterogeneities as well as in the presence of pathological situations, such as myocardial ischemia and infarct scars. However, our results have shown that under idealized and homogeneous conditions the mechanical markers considered do not yield completely reliable estimates of the electrical excitation sequence. Thus, we believe that their performance would even worsen in the presence of heterogeneities, realistic geometries and further complex modeling assumptions. 


\section{Acknowledgments}

This work was partially supported by grants of Istituto di Matematica Applicata e Tecnologie Informatiche IMATI - C.N.R., Pavia, Italy, of Istituto Nazionale di Alta Matematica (INdAM), Italy, and by the ISCRA-C grants iSCAR-HP10CP5Z5I and CEMSim-HP10CS6UNL of CINECA, Italy.

\section{References}

[1] I. Adeniran, J. C. Hancox, and H. Zhang. Effect of cardiac ventricular mechanical contraction on the characteristics of the ECG: A simulation study. J. Biomed. Sci. Eng., 6(12):47-60, 2013.

[2] D. Ambrosi, G. Arioli, F. Nobile, and A. Quarteroni. Electromechanical coupling in cardiac dynamics: The active strain approach. SIAM J. Appl. Math., 71(2):605-621, 2011.

[3] H. Ashikaga, B. A. Coppola, B. Hopenfeld, E. S. Leifer, E. R. McVeigh, and J. H. Omens. Transmural dispersion of myofiber mechanics. J. Am. Coll. Cardiol., 49(8):909-916, 2007.

[4] H. Ashikaga, J. H. Omens, N. B. Ingels, and J. W. Covell. Transmural mechanics at left ventricular epicardial pacing site. Am. J. Physiol. Heart Circ. Physiol., 286(6):H2401-H2407, 2004 .

[5] C. H. Augustijn, F. W. P. T. Arts, and R. S. Reneman. Mapping the sequence of contraction of the canine left ventricle. Pflugers Arch., 419:529-533, 1991.

[6] S. Balay, K. Buschelman, W. D. Gropp, D. Kaushik, M. Knepley, L. C. McInnes, B. F. Smith, and H. Zhang. Petsc users manual. Tech. Rep. ANL-95/11 - Revision 3.3, Argonne National Laboratory, 2012.

[7] P. H. M. Bovendeerd, W. Kroon, and T. Delhaas. Determinants of left ventricular shear strain. Am. J. Physiol. Heart Circ. Physiol., 297(3):H1058-H1068, 2009.

[8] S. G. Campbell, S. N. Flaim, C. H. Leem, and A. D. McCulloch. Mechanisms of transmurally varying myocyte electromechanics in an integrated computational model. Philosophical Transactions of the Royal Society A: Mathematical, Physical and Engineering Sciences, 366(1879):3361-3380, July 2008.

[9] S. G. Campbell, E. Howard, J. Aguado-Sierra, B. A. Coppola, J. H. Omens, L. J. Mulligan, A. D. McCulloch, and R. C. P. Kerckhoffs. Effect of transmurally heterogeneous myocyte excitation-contraction coupling on canine left ventricular electromechanics. Exp. Physiol., 94(5):541-552, 2009.

[10] S. G. Campbell, E. Howard, J. Aguado-Sierra, B. A. Coppola, J. H. Omens, L. J. Mulligan, A. D. McCulloch, and R. C. P. Kerckhoffs. Effect of transmurally heterogeneous myocyte excitation-contraction coupling on canine left ventricular electromechanics. Experimental Physiology, 94(5):541-552, Apr. 2009. 
[11] P. Colli Franzone, L. Guerri, M. Pennacchio, and B. Taccardi. Spread of excitation in 3d models of the anisotropic cardiac tissue. ii. Effects of fiber architecture and ventricular geometry. Math. Biosci., 147:131-171, 1998.

[12] P. Colli Franzone, L. F. Pavarino, and S. Scacchi. Mathematical Cardiac Electrophysiology. Springer, New York, 2014.

[13] P. Colli Franzone, L. F. Pavarino, and S. Scacchi. Parallel multilevel solvers for the cardiac electro-mechanical coupling. Appl. Numer. Math., 95:140-153, 2015.

[14] P. Colli Franzone, L. F. Pavarino, and S. Scacchi. Bioelectrical effects of mechanical feedbacks in a strongly coupled cardiac electro-mechanical model. Math. Mod. Meth. Appl. Sci., 26:27-57, 2016 .

[15] P. Colli Franzone, L. F. Pavarino, and S. Scacchi. Joint influence of transmural heterogeneities and wall deformation on cardiac bioelectrical activity: A simulation study. Math. Biosci., 280:71-86, 2016.

[16] P. Colli Franzone, L. F. Pavarino, and S. Scacchi. Effects of mechanical feedback on the stability of cardiac scroll waves: A bidomain electro-mechanical simulation study. Chaos, 27(9):093905, 2017.

[17] J. Constantino, Y. Hu, and N. A. Trayanova. A computational approach to understanding the cardiac electromechanical activation sequence in the normal and failing heart, with translation to the clinical practice of CRT. Progr. Biophys. Molec. Biol., 110(2-3):372-379, 2012.

[18] J. M. Cordeiro, L. Greene, C. Heilmann, D. Antzelevitch, and C. Antzelevitch. Transmural heterogeneity of calcium activity and mechanical function in the canine left ventricle. American Journal of Physiology-Heart and Circulatory Physiology, 286(4):H1471-H1479, Apr. 2004.

[19] A. Costet, J. Provost, A. Gambhir, Y. Bobkov, P. Danilo, G. J. Boink, M. R. Rosen, and E. E. Konofagou. Electromechanical wave imaging of biologically and electrically paced canine hearts in vivo. Ultrasound Med. Biol., 40(1):177-187, 2014.

[20] F. Del Bianco, P. Colli Franzone, S. Scacchi, and L. Fassina. Electromechanical effects of concentric hypertrophy on the left ventricle: A simulation study. Comput. Biol. Med., 99:236$256,2018$.

[21] T. Delhaas, F. W. P. T. Arts, and R. S. Reneman. Relation between regional electrical activation time and subepicardial fiber strain in the canine left ventricle. Pflugers Arch., 423:78-87, 1993.

[22] D. Durrer, R. T. V. Dam, G. E. Freud, M. J. Janse, F. L. Meijler, and R. C. Arzbaecher. Total excitation of the isolated human heart. Circulation, 41:899-912, 1970.

[23] T. S. E. Eriksson, A. J. Prassl, G. Plank, and G. A. Holzapfel. Influence of myocardial fiber/sheet orientations on left ventricular mechanical contraction. Math. Mech. Solids, 18:592606, 2013.

[24] G. M. Faber and Y. Rudy. Action potential and contractility changes in [na+]i overloaded cardiac myocytes: A simulation study. Biophys. J., 78(5):2392-2404, 2000. 
[25] O. P. Faris, F. J. Evans, D. B. Ennis, P. A. Helm, J. L. Taylor, A. S. Chesnick, M. A. Guttman, C. Ozturk, and E. R. McVeigh. Novel technique for cardiac electromechanical mapping with magnetic resonance imaging tagging and an epicardial electrode sock. Ann. Biomed. Eng., 31(4):430-440, 2003.

[26] L. Fassina, G. Rozzi, S. Rossi, S. Scacchi, M. Galetti, F. P. Lo Muzio, F. Del Bianco, P. Colli Franzone, G. Petrilli, G. Faggian, and M. Miragoli. Cardiac kinematic parameters computed from video of in situ beating heart. Sci. Rep., 7:46143, 2017.

[27] T. Fritz, C. Wieners, G. Seemann, H. Steen, and O. Dössel. Simulation of the contraction of the ventricles in a human heart model including atria and pericardium. Biomech. Model. Mechanobiol., 13(3):627-641, 2014.

[28] L. Gepstein, A. Goldin, J. Lessick, G. Hayam, S. Shpun, Y. Schwartz, G. Hakim, R. Shofty, A. Turgeman, D. Kirshenbaum, and S. A. Ben-Haim. Electromechanical characterization of chronic myocardial infarction in the canine coronary occlusion model. Circulation, 98(19):20552064, 1998.

[29] J. Grondin, A. Costet, E. Bunting, A. Gambhir, H. Garan, E. Wan, and E. E. Konofagou. Validation of electromechanical wave imaging in a canine model during pacing and sinus rhythm. Heart Rhythm, 13(11):2221-2227, 2016.

[30] V. Gurev, J. Constantino, J. Rice, and N. Trayanova. Distribution of electromechanical delay in the heart: Insights from a three-dimensional electromechanical model. Biophys. J., 99(3):745$754,2010$.

[31] A. K. Heikhmakhtiar, A. J. Ryu, E. B. Shim, K.-S. Song, N. A. Trayanova, and K. M. Lim. Influence of LVAD function on mechanical unloading and electromechanical delay: a simulation study. Med. Biol. Eng. Comput., 56(5):911-921, 2018.

[32] G. A. Holzapfel and R. W. Ogden. Constitutive modelling of passive myocardium. a structurally-based framework for material characterization. Phil. Trans. R. Soc. London A, 367:3445-3475, 2009.

[33] P. J. Hunter, A. D. McCulloch, , and H. E. D. J. ter Keurs. Modelling the mechanical properties of cardiac muscle. Progr. Biophys. Molec. Biol., 69:289-331, 1998.

[34] R. H. Keldermann, M. P. Nash, H. Gelderblom, V. Y. Wang, and A. V. Panfilov. Electromechanical wavebreak in a model of the human left ventricle. Am. J. Physiol.-Heart Circ. Physiol., 299(1):H134-H143, 2010.

[35] R. C. P. Kerckhoffs, O. P. Faris, P. H. M. Bovendeerd, F. W. Prinzen, K. Smits, E. R. McVeigh, and T. Arts. Timing of depolarization and contraction in the paced canine left ventricle: Model and experiment. J. Cardiovasc. Electrophysiol., 14(s10):S188-S195, 2003.

[36] R. C. P. Kerckhoffs, O. P. Faris, P. H. M. Bovendeerd, F. W. Prinzen, K. Smits, E. R. McVeigh, and T. Arts. Electromechanics of paced left ventricle simulated by straightforward mathematical model: comparison with experiments. Am. J. Physiol.-Heart Circ. Physiol., 289(5):H1889-H1897, 2005. 
[37] S. Land, S. A. Niederer, J. M. Aronsen, E. K. S. Espe, L. L. Zhang, W. E. Louch, I. Sjaastad, O. M. Sejersted, and N. P. Smith. An analysis of deformation-dependent electromechanical coupling in the mouse heart. J. Physiol., 590:4553-4569, 2012.

[38] M. Landajuela, C. Vergara, A. Gerbi, L. Dedè, L. Formaggia, and A. Quarteroni. Numerical approximation of the electromechanical coupling in the left ventricle with inclusion of the purkinje network. Int. J. Numer. Meth. Biomed. Eng., 34(7):e2984, 2018.

[39] L. C. Lee, J. Sundnes, M. Genet, J. F. Wenk, and S. T. Wall. An integrated electromechanicalgrowth heart model for simulating cardiac therapies. Biomech. Mod. Mechanobiol., 15(4):791$803,2015$.

[40] M. P. Nash and A. V. Panfilov. Electromechanical model of excitable tissue to study reentrant cardiac arrhythmias. Progr. Biophys. Molec. Biol., 85(2-3):501-522, 2004.

[41] S. A. Niederer, P. J. Hunter, and N. P. Smith. A quantitative analysis of cardiac myocyte relaxation: a simulation study. Biophys. J., 90:1697-1722, 2006.

[42] S. A. Niederer and N. P. Smith. A mathematical model of the slow force response to stretch in rat ventricular myocytes. Biophys. J., 92(11):4030-4044, 2007.

[43] S. A. Niederer and N. P. Smith. An improved numerical method for strong coupling of excitation and contraction models in the heart. Progr. Biophys. Molec. Biol., 96:90-111, 2008.

[44] P. Pathmanathan and J. P. Whiteley. A numerical method for cardiac mechanoelectric simulations. Ann. Biomed. Eng., 37(5):860-873, 2009.

[45] L. F. Pavarino and S. Scacchi. Multilevel additive Schwarz preconditioners for the bidomain reaction-diffusion system. SIAM J. Sci. Comp., 31(1):420-443, jan 2008.

[46] L. F. Pavarino, S. Scacchi, and S. Zampini. Newton-Krylov-BDDC solvers for nonlinear cardiac mechanics. Comp. Meth. Appl. Mech. Engrg., 295:562-580, oct 2015.

[47] F. W. Prinzen, C. H. Augustijn, M. A. Allessie, T. Arts, T. Delhaas, and R. S. Reneman. The time sequence of electrical and mechanical activation during spontaneous beating and ectopic stimulation. Eur. Heart J., 13:535-543, 1992.

[48] J. Provost, V. Gurev, N. Trayanova, and E. E. Konofagou. Mapping of cardiac electrical activation with electromechanical wave imaging: An in silico-in vivo reciprocity study. Heart Rhythm, 8(5):752-759, 2011.

[49] J. Provost, W.-N. Lee, K. Fujikura, and E. Konofagou. Electromechanical wave imaging of normal and ischemic hearts in vivo. IEEE Trans. Med. Imag., 29(3):625-635, 2010.

[50] J. Provost, W.-N. Lee, K. Fujikura, and E. E. Konofagou. Imaging the electromechanical activity of the heart in vivo. Proc. Nat. Acad. Sci., 108(21):8565-8570, 2011.

[51] A. Quarteroni, A. Manzoni, and C. Vergara. The cardiovascular system: mathematical modelling, numerical algorithms and clinical applications. Acta Numerica, 26:365-590, 2017. 
[52] J. J. Rice, F. Wang, D. M. Bers, and P. P. de Tombe. Approximate model of cooperative activation and cross bridge cycling in cardiac muscle using ordinary differential equations. Biophys. J., 95:2368-2390, 2008.

[53] K. Russell, A. Opdahl, E. W. Remme, O. Gjesdal, H. Skulstad, E. Kongsgaard, T. Edvardsen, and O. A. Smiseth. Evaluation of left ventricular dyssynchrony by onset of active myocardial force generation. Circulation: Cardiovasc. Imag., 3(4):405-414, 2010.

[54] K. Russell, O. A. Smiseth, O. Gjesdal, E. Qvigstad, P. A. Norseng, I. Sjaastad, A. Opdahl, H. Skulstad, T. Edvardsen, and E. W. Remme. Mechanism of prolonged electromechanical delay in late activated myocardium during left bundle branch block. Am. J. Physiol. Heart Circ. Physiol., 301(6):H2334-H2343, 2011.

[55] S. Scacchi. A hybrid multilevel Schwarz method for the bidomain model. Comp. Meth. Appl. Mech. Engrg., 197(45-48):4051-4061, aug 2008.

[56] A. Scatteia, A. Baritussio, and C. Bucciarelli-Ducci. Strain imaging using cardiac magnetic resonance. Heart Failure Rev., 22(4):465-476, 2017.

[57] P. P. Sengupta, B. K. Khandheria, J. Korinek, J. Wang, A. Jahangir, J. B. Seward, and M. Belohlavek. Apex-to-base dispersion in regional timing of left ventricular shortening and lengthening. J. Am. Coll. Cardiol., 47(1):163-172, 2006.

[58] J. Sundnes, S. Wall, H. Osnes, T. Thorvaldsen, and A. McCulloch. Improved discretisation and linearisation of active tension in strongly coupled cardiac electro-mechanics simulations. Comput. Meth. Biomech. Biomed. Eng., 17(6):604-615, 2014.

[59] T. P. Usyk, R. Mazhari, and A. D. McCulloch. Effect of laminar orthotropic myofiber architecture on regional stress and strain in the canine left ventricle. J. Elast. Phys. Sci. Sol., 61(1/3):143-164, 2000.

[60] T. P. Usyk and A. D. McCulloch. Relationship between regional shortening and asynchronous electrical activation in a three-dimensional model of ventricular electromechanics. J. Cardiovasc. Electrophysiol., 14(s10):S196-S202, 2003.

[61] S. T. Wall, J. M. Guccione, M. B. Ratcliffe, and J. S. Sundnes. Electromechanical feedback with reduced cellular connectivity alters electrical activity in an infarct injured left ventricle: a finite element model study. Am. J. Physiol.-Heart Circ. Physiol., 302(1):H206-H214, 2012.

[62] Y. Wang, P. S. Cuculich, J. Zhang, K. A. Desouza, R. Vijayakumar, J. Chen, M. N. Faddis, B. D. Lindsay, T. W. Smith, and Y. Rudy. Noninvasive electroanatomic mapping of human ventricular arrhythmias with electrocardiographic imaging. Science Transl. Med., 3(98):98ra8498ra84, 2011.

[63] B. T. Wyman, W. C. Hunter, F. W. Prinzen, O. P. Faris, and E. R. McVeigh. Effects of singleand biventricular pacing on temporal and spatial dynamics of ventricular contraction. Am. J. Physiol. Heart Circ. Physiol., 282(1):H372-H379, 2002.

[64] B. T. Wyman, W. C. Hunter, F. W. Prinzen, and E. R. McVeigh. Mapping propagation of mechanical activation in the paced heart with MRI tagging. Am. J. Physiol. Heart Circ. Physiol., 276(3):H881-H891, 1999. 
[65] J. J. M. Zwanenburg, M. J. W. Götte, J. P. A. Kuijer, R. M. Heethaar, A. C. van Rossum, and J. T. Marcus. Timing of cardiac contraction in humans mapped by high-temporal-resolution MRI tagging: early onset and late peak of shortening in lateral wall. Am. J. Physiol. Heart Circ. Physiol., 286(5):H1872-H1880, 2004. 

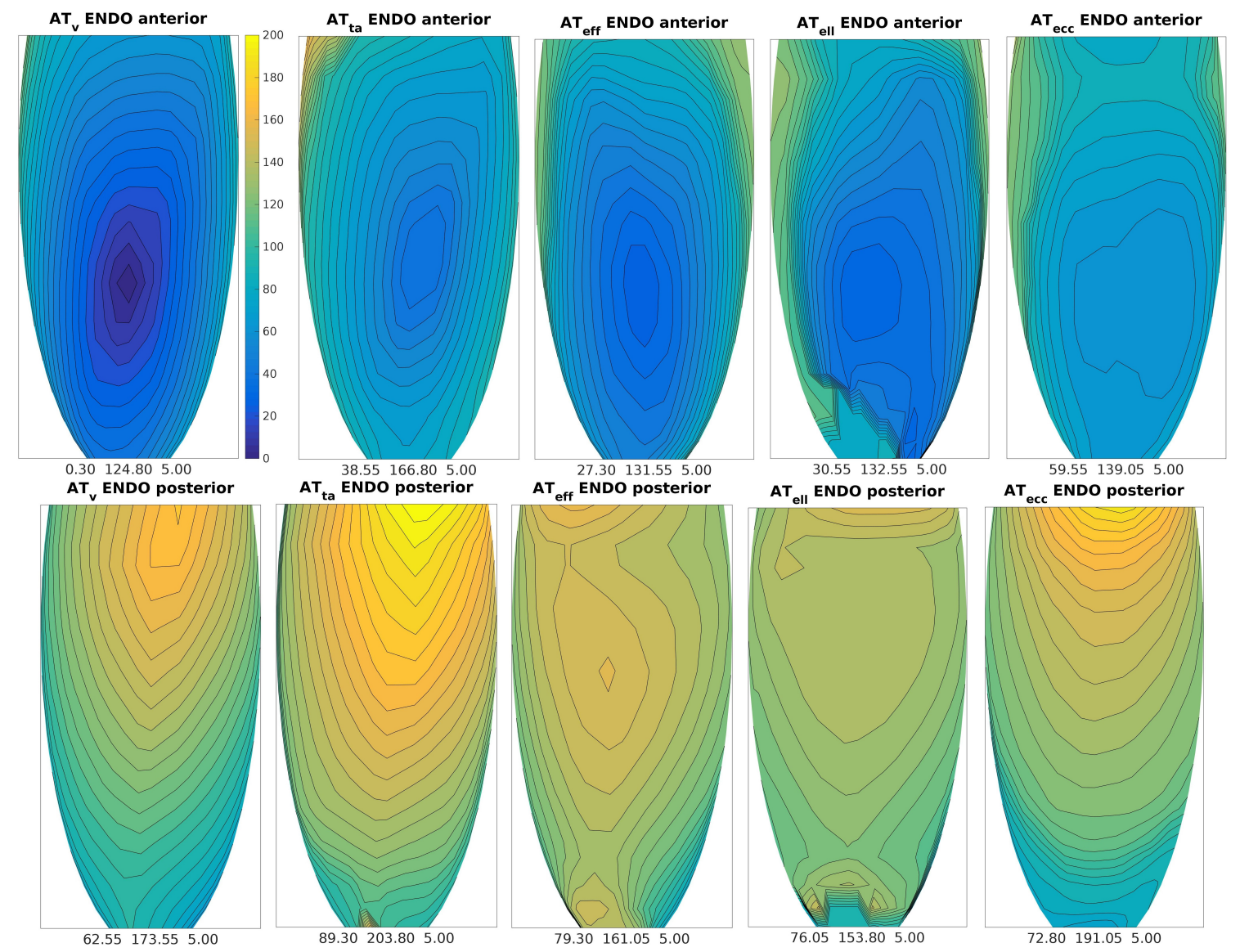

Figure 3: Ectopic endocardial stimulation. $\mathrm{AT}_{v}, \mathrm{AT}_{t a}, \mathrm{AT}_{\text {eff }}, \mathrm{AT}_{\text {ell }}$ and $\mathrm{AT}_{\text {ecc }}$ endocardial distributions, anterior and posterior view. Below each panel are reported the minimum, maximum and step (all in $m s$ ) of the displayed map. 

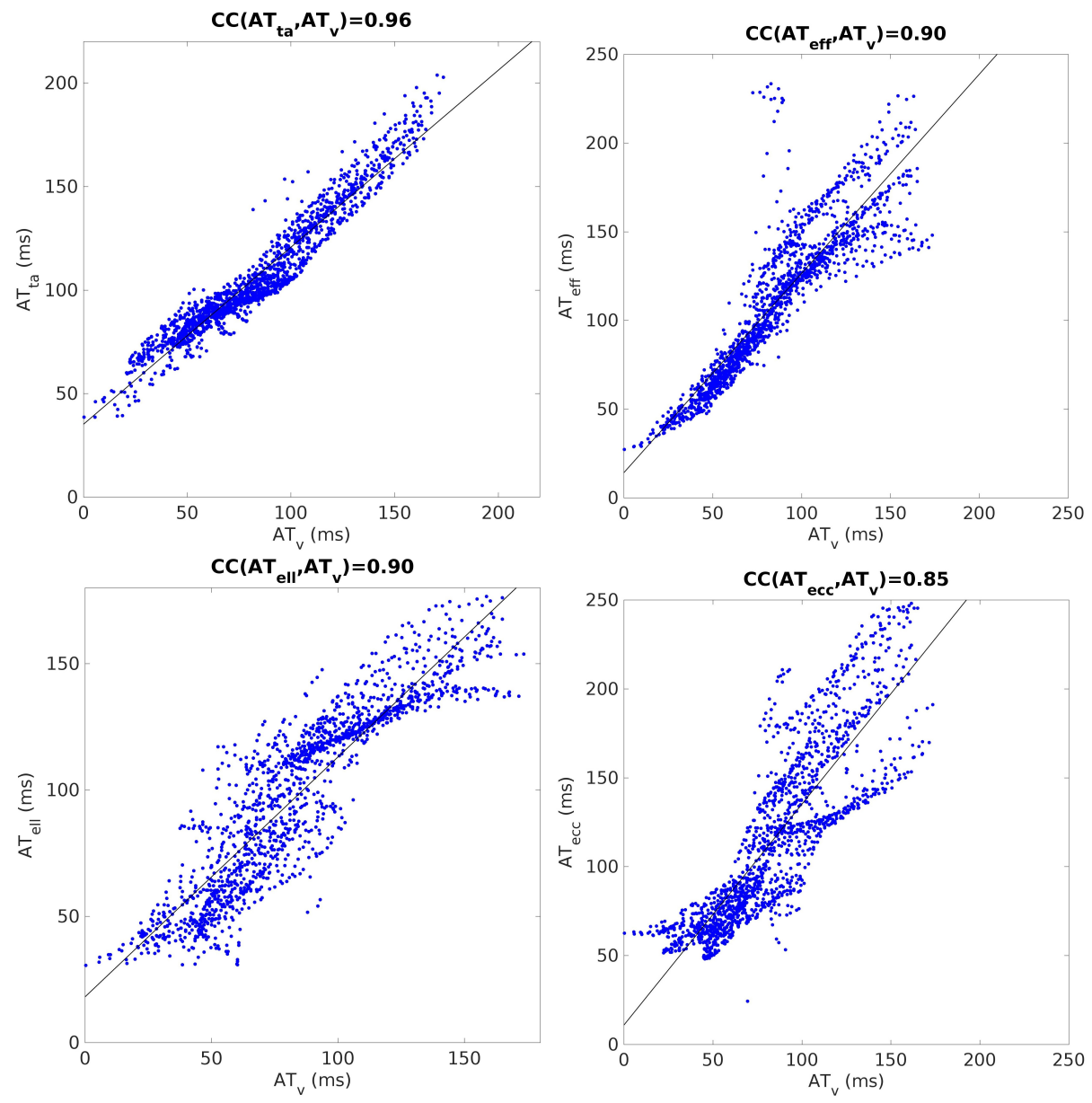

Figure 4: Ectopic endocardial stimulation. Regression plots. Each panel reports the correlation coefficient $\mathrm{CC}\left(\mathrm{AT}_{s}, \mathrm{AT}_{v}\right)$ on the entire ventricular volume for the mechanical activation markers $s=t a$, eff, ecc. 

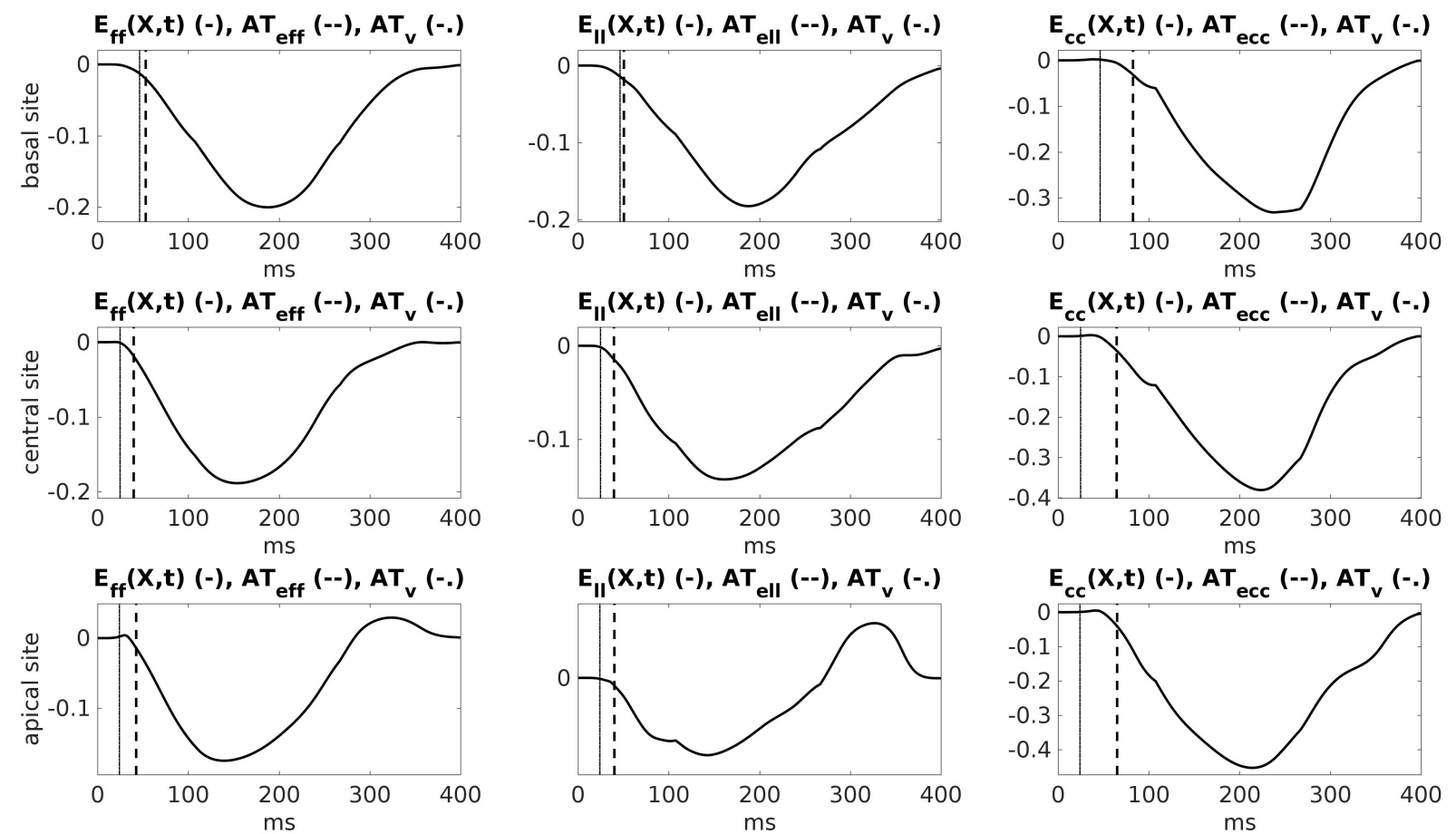

Figure 5: Ectopic endocardial stimulation. Strain waveforms with associated mechanical activation markers at three selected endocardial anterior sites located in the basal region (first row), central region (second row) and apical region (third row). 

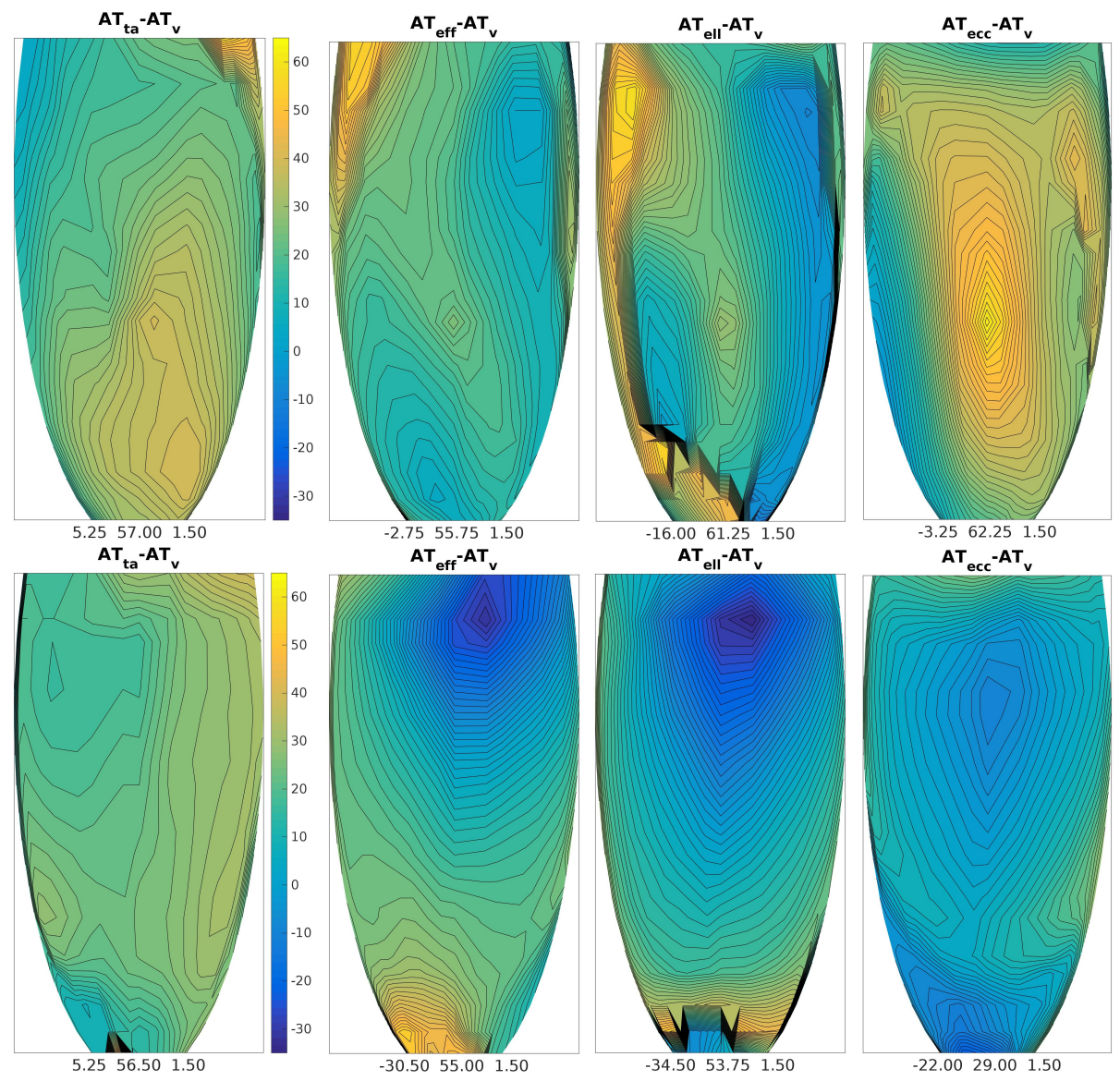

Figure 6: Ectopic endocardial stimulation. Endocardial distributions, anterior and posterior view, of the electro-mechanical delays $\mathrm{AT}_{t a}-\mathrm{AT}_{v}, \mathrm{AT}_{e f f}-\mathrm{AT}_{v}, \mathrm{AT}_{e l l}-\mathrm{AT}_{v}$ and $\mathrm{AT}_{e c c}-\mathrm{AT}_{v}$. Below each panel are reported the minimum, maximum and step (all in $m s$ ) of the displayed map. 

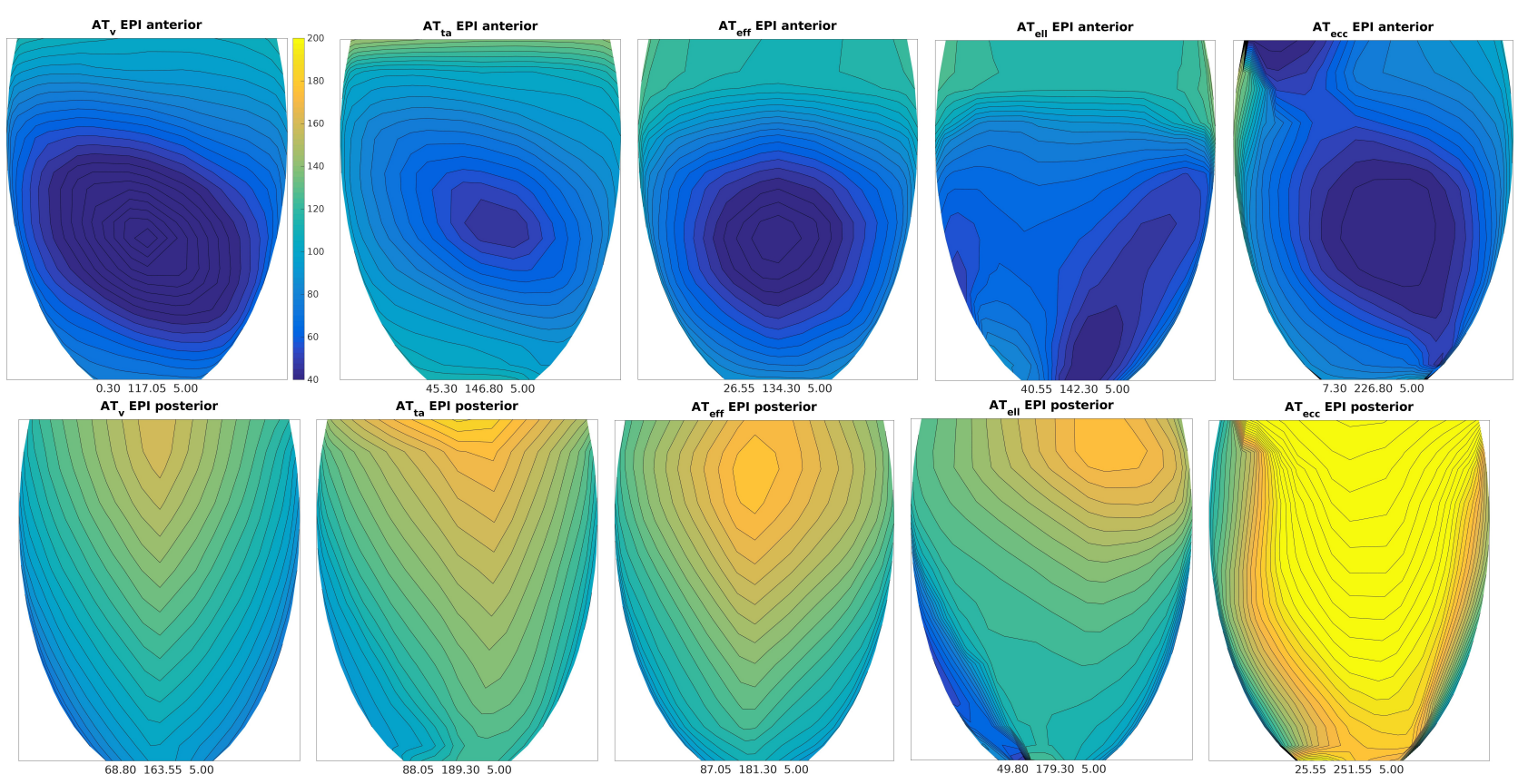

Figure 7: Ectopic epicardial stimulation. $\mathrm{AT}_{v}, \mathrm{AT}_{t a}, \mathrm{AT}_{\text {eff }}, \mathrm{AT}_{\text {ell }}$ and $\mathrm{AT}_{\text {ecc }}$ epicardial distributions, anterior and posterior view. Same format as in Fig. 3. 

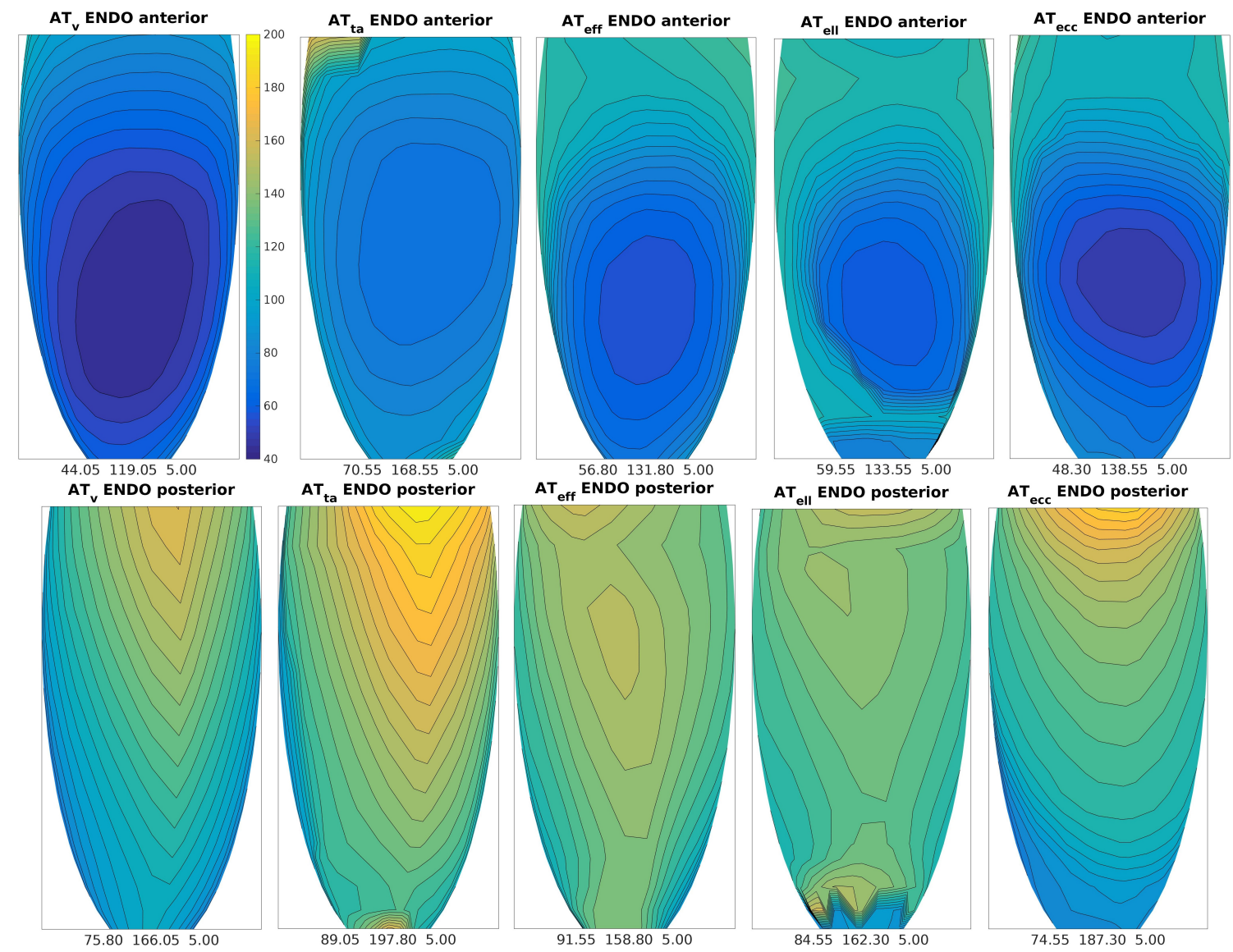

Figure 8: Ectopic epicardial stimulation. $\mathrm{AT}_{v}, \mathrm{AT}_{t a}, \mathrm{AT}_{e f f}, \mathrm{AT}_{\text {ell }}$ and $\mathrm{AT}_{\text {ecc }}$ endocardial distributions, anterior and posterior view. Same format as in Fig. 3. 

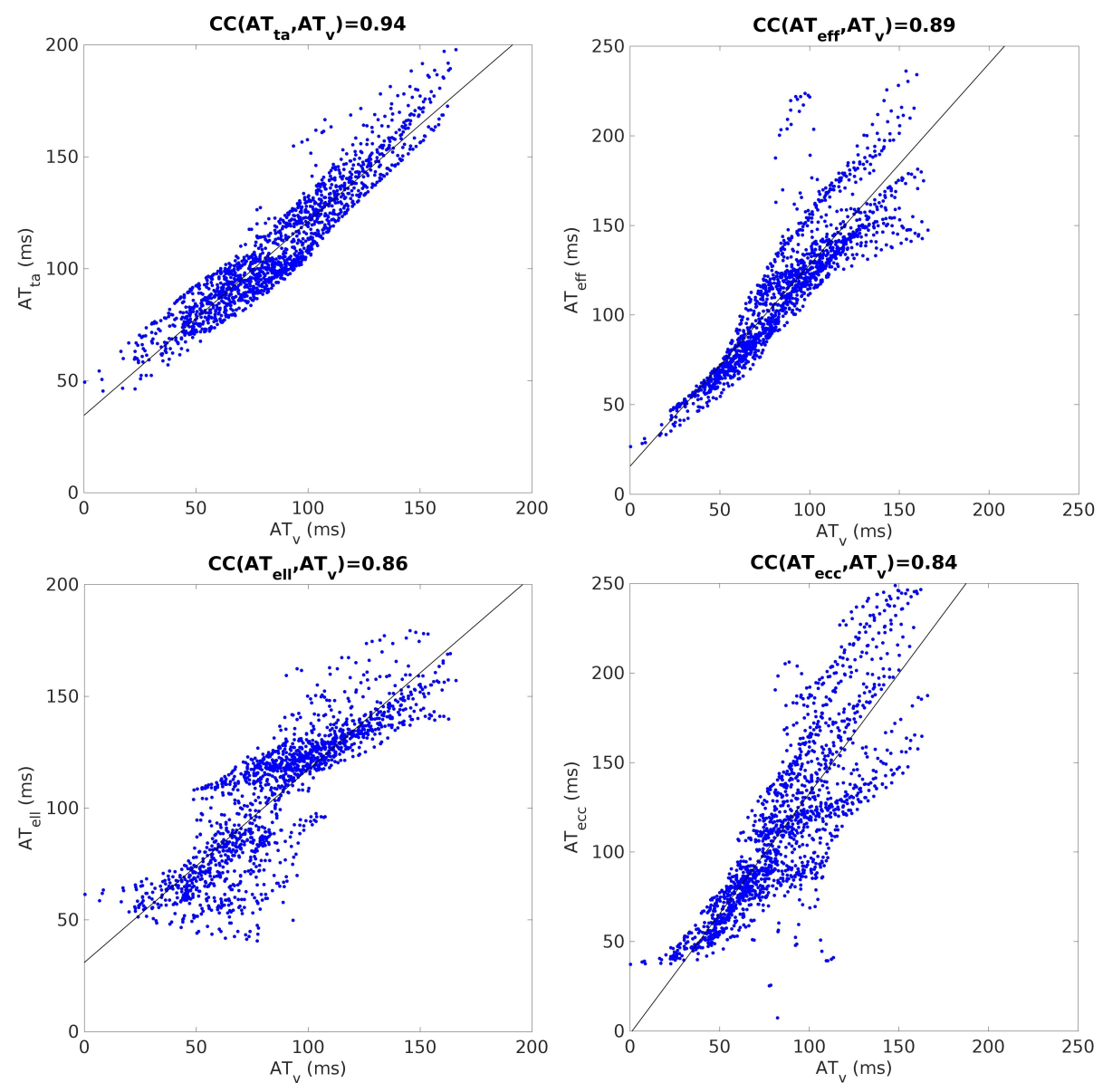

Figure 9: Ectopic epicardial stimulation. Regression plots. Same format as in Fig. 4. 

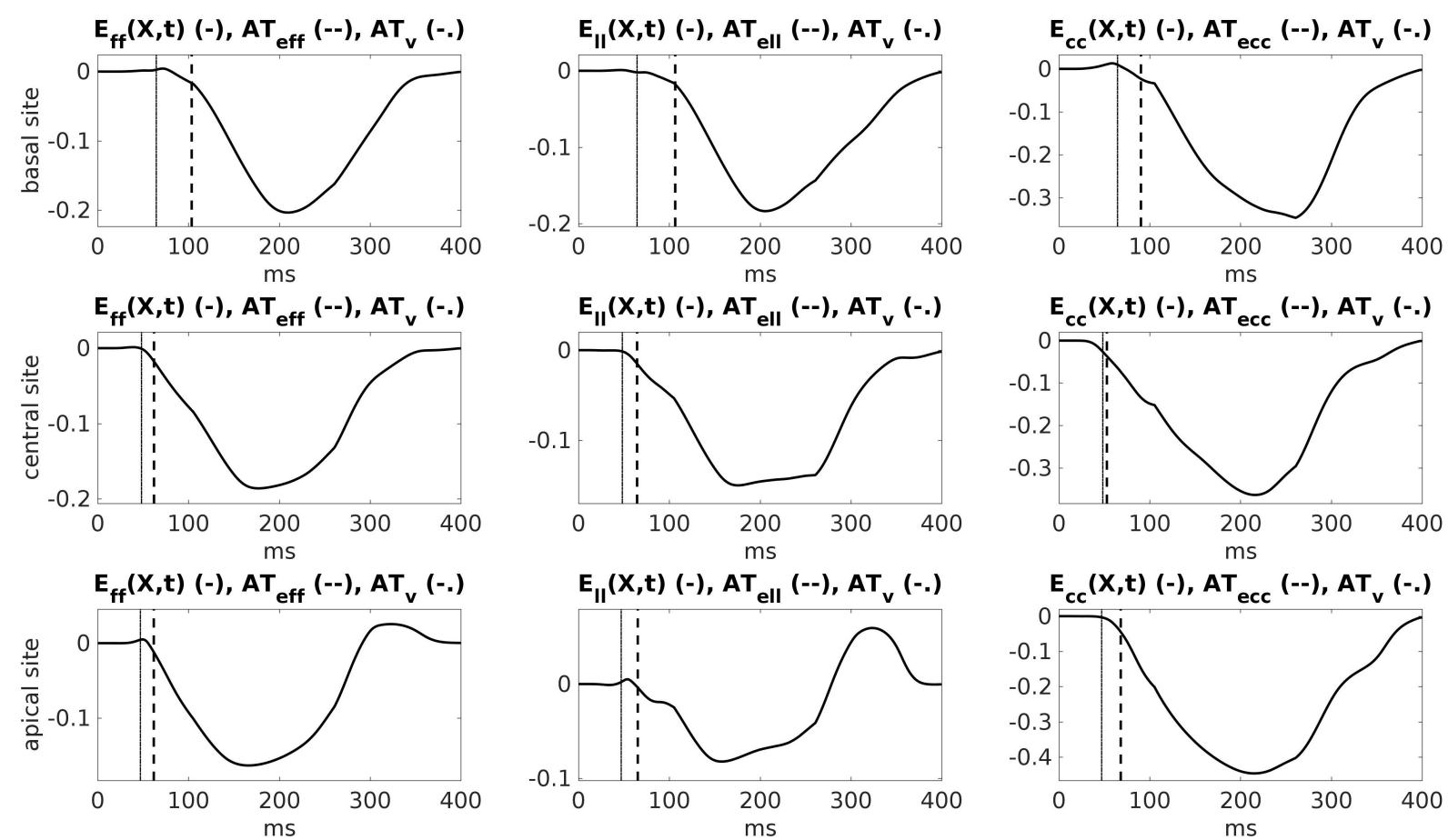

Figure 10: Ectopic epicardial stimulation. Strain waveforms with associated mechanical activation markers at three selected endocardial anterior sites located in the basal region (first row), central region (second row) and apical region (third row). 

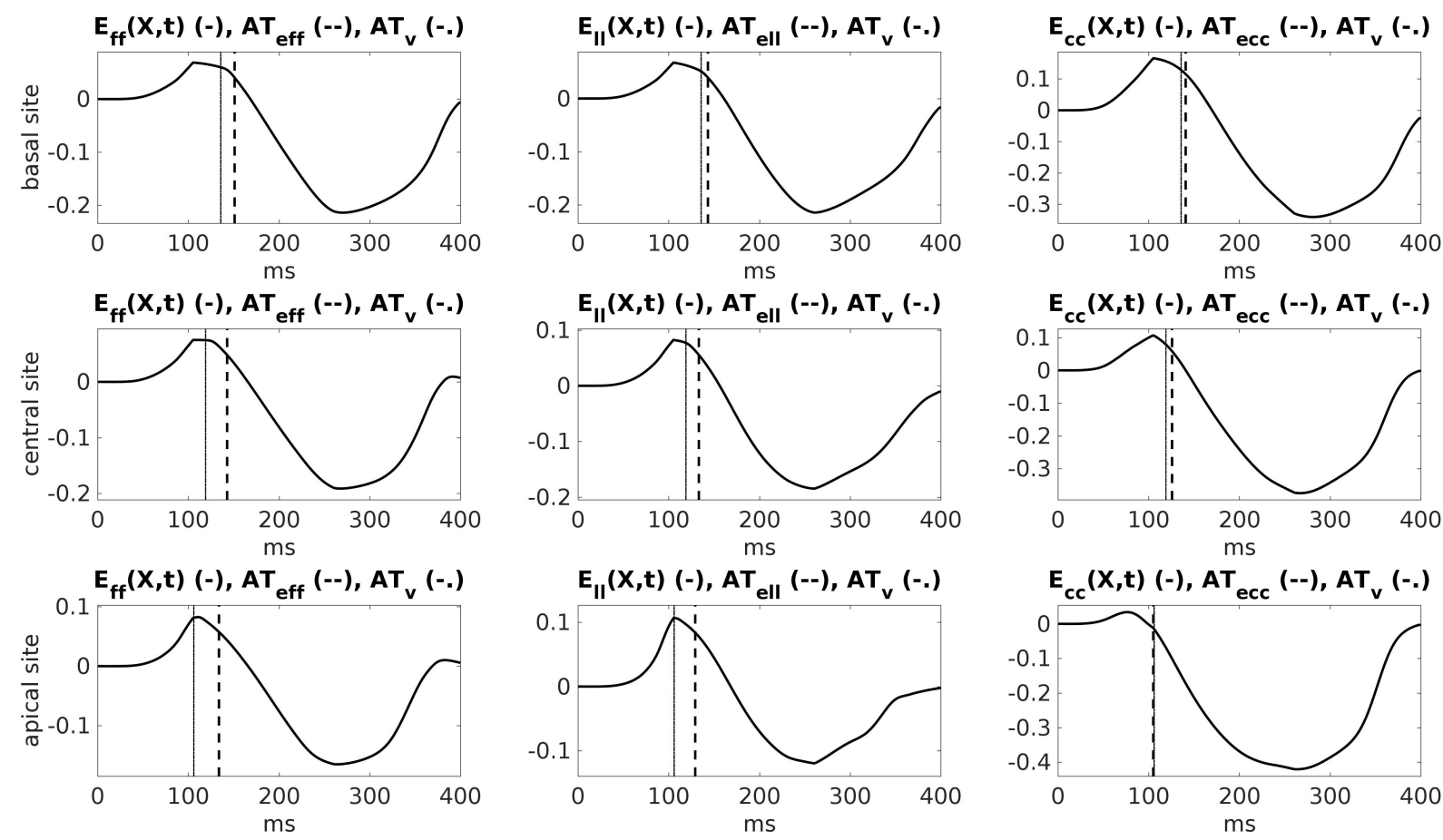

Figure 11: Ectopic epicardial stimulation. Strain waveforms with associated mechanical activation markers at three selected endocardial posterior sites located in the basal region (first row), central region (second row) and apical region (third row). 

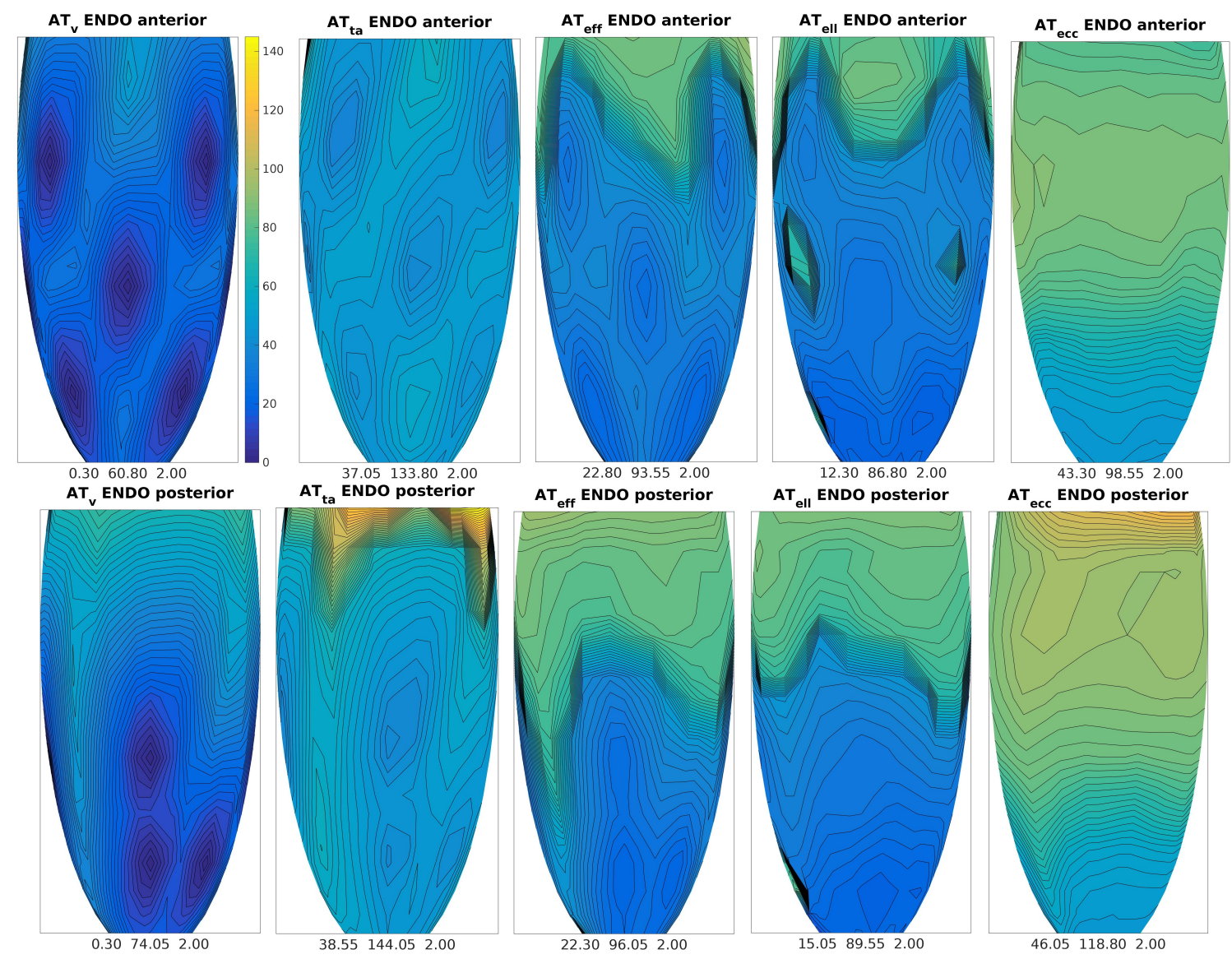

Figure 12: Multiple endocardial stimulations. $\mathrm{AT}_{v}, \mathrm{AT}_{t a}, \mathrm{AT}_{\text {eff }}, \mathrm{AT}_{\text {ell }}$ and $\mathrm{AT}_{\text {ecc }}$ endocardial distributions, anterior and posterior view. Same format as in Fig. 3. 

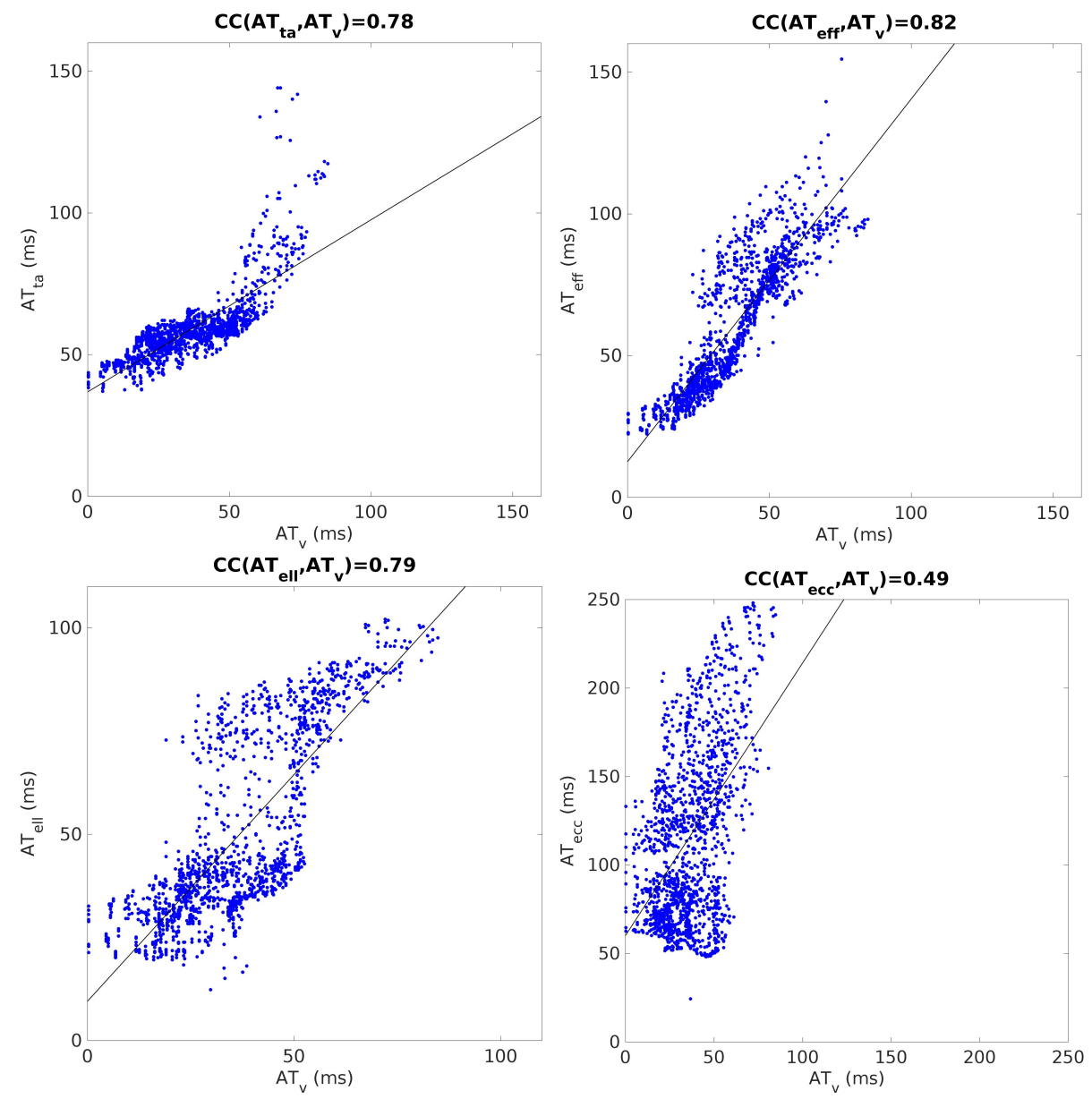

Figure 13: Multiple endocardial stimulations. Regression plots. Same format as in Fig. 4. 

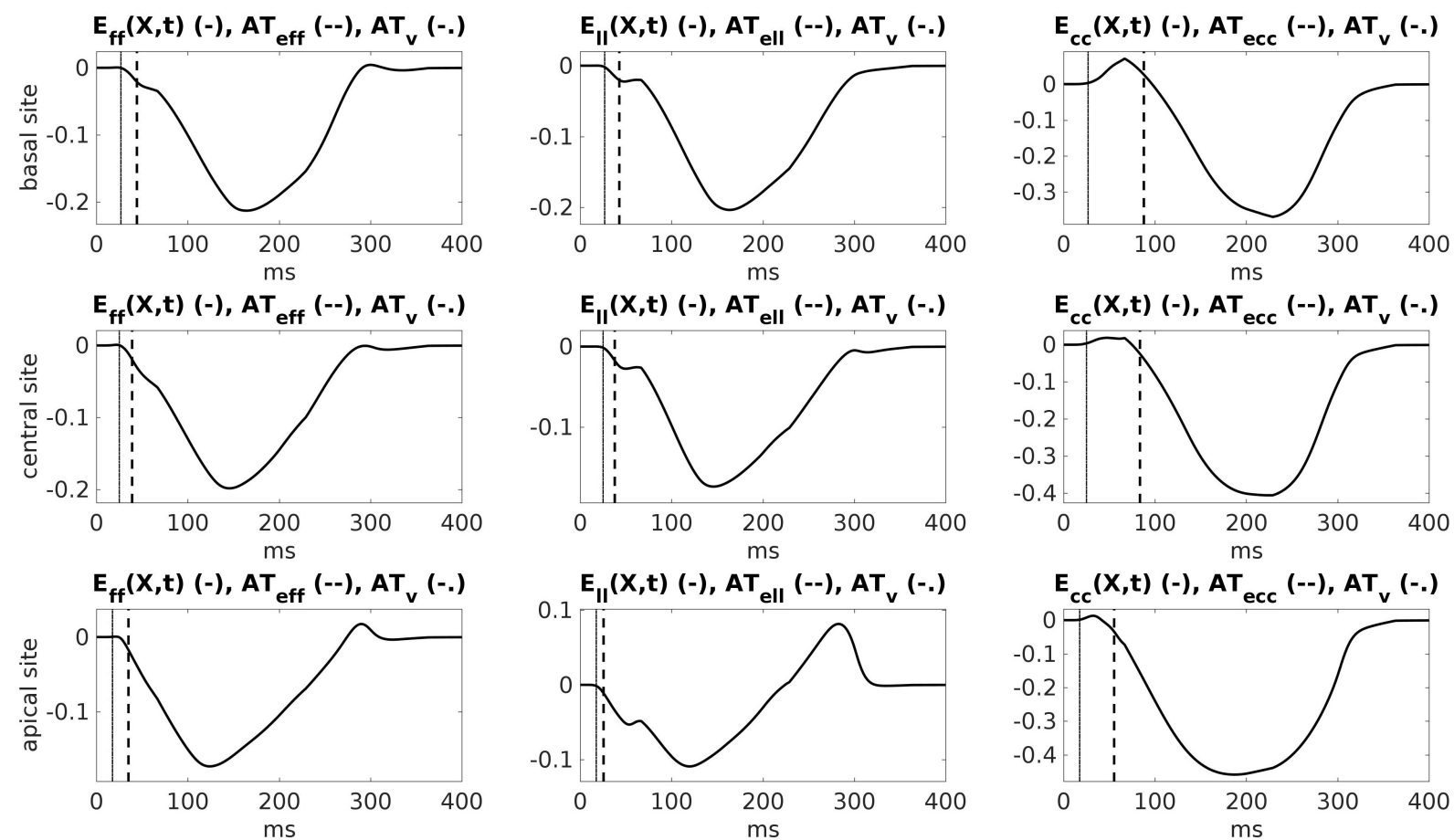

Figure 14: Multiple endocardial stimulations. Strain waveforms with associated mechanical activation markers at three selected endocardial anterior sites located in the basal region (first row), central region (second row) and apical region (third row). 